\title{
Three dimensional nonlinear magnetic AdS solutions through topological defects
}

\author{
S. H. Hendi ${ }^{1,2, a} \mathbb{C}$, B. Eslam Panah ${ }^{1, b}$, M. Momennia ${ }^{1, \mathrm{c}}$, S. Panahiyan ${ }^{1, \mathrm{~d}}$ \\ ${ }^{1}$ Physics Department and Biruni Observatory, College of Sciences, Shiraz University, 71454 Shiraz, Iran \\ ${ }^{2}$ Research Institute for Astronomy and Astrophysics of Maragha (RIAAM), Maragha, Iran
}

Received: 8 June 2015 / Accepted: 10 September 2015 / Published online: 28 September 2015

(C) The Author(s) 2015. This article is published with open access at Springerlink.com

\begin{abstract}
Inspired by large applications of topological defects in describing different phenomena in physics, and considering the importance of three dimensional solutions in AdS/CFT correspondence, in this paper we obtain magnetic anti-de Sitter solutions of nonlinear electromagnetic fields. We take into account three classes of nonlinear electrodynamic models; first two classes are the well-known BornInfeld like models including logarithmic and exponential forms and third class is known as the power Maxwell invariant nonlinear electrodynamics. We investigate the effects of these nonlinear sources on three dimensional magnetic solutions. We show that these asymptotical AdS solutions do not have any curvature singularity and horizon. We also generalize the static metric to the case of rotating solutions and find that the value of the electric charge depends on the rotation parameter. Finally, we consider the quadratic Maxwell invariant as a correction of Maxwell theory and we investigate the effects of nonlinearity as a correction. We study the behavior of the deficit angle in presence of these theories of nonlinearity and compare them with each other. We also show that some cases with negative deficit angle exists which are representing objects with different geometrical structure. We also show that in case of the static only magnetic field exists whereas by boosting the metric to rotating one, electric field appears too.
\end{abstract}

\section{Introduction}

Topological defects are playing crucial role in studying different physical phenomena in context of quantum theory, condensed matter, cosmology and string theory. It was shown

\footnotetext{
a e-mail: hendi@shirazu.ac.ir

be-mail: behzad_eslampanah@yahoo.com

ce-mail: momennia1988@gmail.com

de-mail: ziexify@gmail.com
}

that, in studying liquid crystal, broad range of phenomena from structural properties to phase transitions are governed by the existence of these topological defects [1-3]. In addition by employing rules governed by these topological defects some experimental modifications were done which lead to improvement of physical insight regarding quantum loops and the quality of obtained materials in different aspects [1-4]. These studies are acceptable in context of condensed matter with ordered media [5]. Another application of these defects is in studying magnetism and nanomagnetism which was done in literature [6,7]. In addition, it is notable that this mathematical tool was also employed in studying vortices in superfluid [8] and Bose-Einstein condensate [9-11]. Also, in studying the phase transition and critical behavior of Bose gas [12], it was used widely. Furthermore, it is worthwhile to mention that these topological defects are essential tools in order to study superconductors and their phase transitions [13-16].

In general a topological defect or topological solution is formed because of symmetry breakdown. Due to different symmetry breakdowns and their dimensionality, the topological defects could be interpreted as different types of known defects. In cosmology, the well-known topological defects are cosmic string (one dimensional lines with one axial or cylindrical symmetry broken), domain walls (two dimensional membranes that form when a discrete symmetry is broken at a phase transition. These walls resemble the walls of a closed-cell foam, dividing the universe into discrete cells), monopoles (cube-like defects that form when a spherical symmetry is broken and are predicted to have magnetic charge, either north or south. The most wellknown monopole is magnetic monopole), textures (form when larger, more complicated symmetry groups are completely broken. They are not as localized as the other defects and are unstable. Other more complex hybrids of these defect types are also possible) and (higher dimensional) branes (for more details see [17]). Certain theories of GUT pre- 
dict that these cosmological defects are formed during phase transition of early Universe [18-24] and they can be used to describe the large scale structure of the Universe. As for cosmic string, asymptotically AdS spacetimes generated by static and spinning magnetic sources in the EinsteinMaxwell gravity have been investigated in [25-27]. The properties and interaction of the superconducting cosmic strings with astrophysical magnetic fields have been studied in [28-30]. Also, superconducting cosmic strings have been investigated in dilaton gravity [31-33], and in BransDicke theory [34]. These solutions are not black holes, and represent spacetimes with conic singularities. From cosmological point of view, the properties of the magnetic (cosmic) string have been studied in many literatures (for e.g., see [3537]). Properties of the QCD static strings and applications of magnetic strings in quantum theories have been explored in [38-43], respectively. The main reason to consider the magnetic string solutions is that they may be interpreted as cosmic strings. As for the domain walls, it has been studied in many literature [44-46] and for textures some studies have been done $[47,48]$. In gravitational point of view, these topological defects are characterized by their masses, rotation parameters and deficit angles, while from cosmological aspect, these topological defects, as predicted, have no gravitational potential and the only property that makes them visible or detectable is their deficit angle which is acting as a cosmological lens [49]. The projection of photon on the surface of these topological defects is modified due to existence of deficit angle. Therefore, in case of these cosmological objects, the property known as deficit angle is so important.

Motivated by the above statements, in this paper we study three dimensional AdS magnetic solutions in context of topological defects in the presence of different nonlinear theories of electrodynamics. As we will see, these solutions do not have any singularity and horizon. Therefore, these solutions are not interpreted as black holes, but spacetimes with a conical singularity. In other words, our solutions are the nonlinear counterparts of the static Einstein-Maxwell-AdS solutions found in Ref. [50-52] and of the rotating solutions in Ref. [53-55].

Most of physical systems have a nonlinear behavior in nature, so, the nonlinear field theories are interesting in physics. The basic motivation for studying the nonlinear electrodynamics (NED) comes from the fact that these theories are generalizations of the Maxwell field and in the special case (weak nonlinearity) they can reduce to the linear Maxwell theory. Other motivations of considering NED are limitations of the Maxwell theory [56,57], description of the self-interaction of virtual electron-positron pairs [58-60] and the radiation propagation inside specific materials [61-64]. Besides, NED improves the basic concept of gravitational redshift and its dependency of any background magnetic field as compared to the well-established method introduced by standard general relativity. In addition, it was recently shown that NED objects can remove both of the big bang and black hole singularities [65-70]. Moreover, from astrophysical point of view, one finds the effects of NED become indeed quite important in superstrong magnetized compact objects, such as pulsars, and particular neutron stars [71-73].

It is well-known that the electric field of a point-like charge has a singularity in its location (origin). In order to remove this divergency, Born and Infeld introduced a NED which is known as Born-Infeld nonlinear electrodynamics (BI NED) theory [74]. After that Soleng and Hendi introduced two different types of BI type NED in [75,76], respectively, which can also remove the electric field divergency of point-like charges near the origin. Soleng's Lagrangian has a logarithmic form and, like BI NED, removes divergences in the electric field, while Hendi's Lagrangian has an exponential form and does not cancel the origin divergency of the electric field but its singularity is much weaker than Einstein-Maxwell theory. Another example of the nonlinear electromagnetic field is power Maxwell invariant (PMI NED) field [77-82]. In general BI-types of NED theories have interesting properties that make them different comparing to other theories of nonlinearity [83-86]. In addition in context of heterotic string theory in low energy limit, the Lagrangian of these kinds of nonlinear theories may rise which gives another strong motivation for considering these theories.

On the other hand, one of the main reasons to consider the $(2+1)$-dimensional solutions comes from the fact that these solutions help us to find a profound insight in the physics of $(2+1)$-dimensional objects and also, play an important role to improve our understanding of gravitational interaction in low-dimensional manifolds. Due to these facts, investigation of $(2+1)$-dimensional spacetimes is important. Three dimensional solutions of black holes and magnetic solutions have been studied by many authors [50-55,87-93]. This tremendous interest in these solutions is due to fact that three dimensional solutions contribute fundamentally to conceptual issues of astrophysical subjects such as black holes thermodynamics [94-96]. In addition, in context of quantum gravity and string theory, also due to AdS/CFT correspondence, these theories play an undeniable important role in describing different phenomena specially in low dimensional spacetime [97-99]. Recently, some theories have been proposed for obtaining magnetic solutions in three dimensions and magnetic monopole $[100,101]$.

The outline of our paper is as follows. In the next section, we give a brief review of the basic field equations of Einstein gravity in the presence of cosmological constant and nonlinear electrodynamics. In Sect. 3, we consider the $(2+1)$-dimensional horizonless metric and obtain magnetic solutions for various sources and investigate its geometric properties. Also, we apply the rotation boost to the static met- 
ric and obtain the conserved quantities of rotating spacetime. Next, we consider nonlinearity as a correction to Maxwell theory and study the magnetic solutions for this case. Geometrical properties of solution will be studied. We finish our paper with some concluding remarks.

\section{Basic field equations}

The $(2+1)$-dimensional action in Einstein gravity with negative cosmological constant $(\Lambda)$ that coupled to nonlinear electrodynamics is given by

$$
\begin{aligned}
\mathcal{I}_{G}= & -\frac{1}{16 \pi} \int_{\mathcal{M}} \mathrm{d}^{3} x \sqrt{-g}\left[\mathcal{R}-2 \Lambda+\mathcal{L}_{N L}(\mathcal{F})\right] \\
& -\frac{1}{8 \pi} \int_{\partial \mathcal{M}} \mathrm{d}^{2} x \sqrt{-\gamma} \Theta(\gamma),
\end{aligned}
$$

where $\mathcal{R}$ is the scalar curvature and $\mathcal{L}_{N L}(\mathcal{F})$ is an arbitrary Lagrangian of nonlinear electrodynamics. The last term in the present equation is the Gibbons-Hawking surface term in which we must add it to the action for a well-defined variational principle [102,103]. In this term $\gamma$ and $\Theta$ are, respectively, the trace of the induced metric $\gamma_{i j}$ and the extrinsic curvature $\Theta_{i j}$ on the boundary $\partial \mathcal{M}$. Varying the action (1) with respect to the gravitational field $g_{\mu \nu}$ and the gauge potential $A_{\mu}$, the field equations are obtained as

$R_{\mu \nu}-\frac{1}{2} g_{\mu \nu}(\mathcal{R}-2 \Lambda)=T_{\mu \nu}$,

$\partial_{\mu}\left(\sqrt{-g} \mathcal{L}_{\mathcal{F}} F^{\mu v}\right)=0$,

where $\mathcal{L}_{\mathcal{F}}=\mathrm{d} \mathcal{L}_{N L}(\mathcal{F}) / \mathrm{d} \mathcal{F}$ and $\mathcal{F}=F_{\mu \nu} F^{\mu \nu}$ is the Maxwell invariant where $F_{\mu \nu}$ is the electromagnetic tensor field. In the presence of nonlinear electromagnetic field, the energy-momentum tensor of Eq. (2) is

$T_{\mu \nu}=\frac{1}{2} g_{\mu \nu} \mathcal{L}_{N L}(\mathcal{F})-2 \mathcal{L}_{\mathcal{F}} F_{\mu \lambda} F_{\nu}^{\lambda}$.

In general the action $\mathcal{I}_{G}$ diverges when evaluated on the solutions, as the Hamiltonian and other associated conserved quantities. Rather than eliminating these divergences by incorporating reference term, a counterterm action $\mathcal{I}_{\text {ct }}$ is added to the action which is functional of the boundary curvature invariants. For asymptotically AdS solutions, one can instead deal with these divergences via the counterterm method inspired by AdS/CFT correspondence [104-109]. We assume that the suitable counterterm is

$\mathcal{I}_{\mathrm{ct}}=-\frac{1}{8 \pi} \int_{\partial \mathcal{M}} \mathrm{d}^{2} x \sqrt{-\gamma} \mathcal{L}_{\mathrm{ct}}$,

where $\mathcal{L}_{\text {ct }}$ is the counterterm Lagrangian and by use of the suitable Lagrangian we will be able to compute the finite conserved quantities. Therefore, the total finite action, $\mathcal{I}$, can be written as

$\mathcal{I}=\mathcal{I}_{G}+\mathcal{I}_{\text {ct }}$.

Having the total finite action, one can use Brown and York definition [110] to construct a divergence free stress-energy tensor as

$T^{\mu \nu}=\frac{1}{8 \pi}\left(\Theta^{\mu \nu}-\Theta \gamma^{\mu \nu}+2 \frac{\delta \mathcal{L}_{\mathrm{ct}}}{\delta \gamma_{\mu \nu}}\right)$.

To compute the conserved charges of a rotating spacetime, we choose a spacelike surface $\mathcal{B}$ in $\partial \mathcal{M}$ with metric $\sigma$, and write the boundary metric in Arnowitt-Deser-Misner (ADM) form

$\gamma_{\mu \nu} \mathrm{d} x^{\mu} \mathrm{d} x^{\nu}=-N^{2} \mathrm{~d} t^{2}+\sigma(\mathrm{d} \varphi+V \mathrm{~d} t)^{2}$,

where the coordinates $\varphi$ is the angular variables parameterizing the hypersurface of constant radial coordinate around the origin, and $N$ and $V$ are the lapse and shift functions, respectively. Considering a Killing vector field $\xi$ on the boundary, then the quasilocal conserved quantities associated with the stress energy momentum tensor of Eq. (7) can be written as

$Q(\xi)=\int_{\mathcal{B}} \mathrm{d} \varphi \sqrt{\sigma} T_{\mu \nu} n^{\mu} \xi^{\nu}$,

where $n^{\mu}$ is the timelike unit normal vector to the boundary $\mathcal{B}$. For boundaries with timelike $(\xi=\partial / \partial t)$ and rotational $(\varsigma=\partial / \partial \varphi)$ Killing vector fields, one can obtain associated conserved quantities in the following forms

$M=\int_{\mathcal{B}} \mathrm{d} \varphi \sqrt{\sigma} T_{\mu \nu} n^{\mu} \xi^{\nu}$,

$J=\int_{\mathcal{B}} \mathrm{d} \varphi \sqrt{\sigma} T_{\mu \nu} n^{\mu} \varsigma^{\nu}$,

provided the surface $\mathcal{B}$ contains the orbits of $\varsigma$. These quantities are the mass and angular momentum of the system enclosed by the boundary $\mathcal{B}$, respectively.

\section{Magnetic solutions with nonlinear sources}

In this section we want to obtain the three dimensional solutions of Eqs. (2)-(4) with considering different electrodynamic models. We consider the following ansatz for the metric

$\mathrm{d} s^{2}=-\frac{\rho^{2}}{l^{2}} \mathrm{~d} t^{2}+\frac{\mathrm{d} \rho^{2}}{g(\rho)}+l^{2} g(\rho) \mathrm{d} \varphi^{2}$, 
where $g(\rho)$ is an arbitrary function of radial coordinate, $\rho$, and should be determined and $l$ is a scale length factor which is related to $\Lambda$. The angular coordinate $\varphi$ is dimensionless and ranges in $0 \leq \varphi<2 \pi$. The motivation for this curious choice for the metric gauge $\left[g_{t t} \propto-\rho^{2}\right.$ and $\left(g_{\rho \rho}\right)^{-1} \propto$ $\left.g_{\varphi \varphi}\right]$ instead of the usual Schwarzschild like $\left[\left(g_{\rho \rho}\right)^{-1} \propto g_{t t}\right.$ and $g_{\varphi \varphi} \propto \rho^{2}$ ] comes from the fact that we are looking for magnetic solutions. It is easy to show that, using a suitable transformation, the metric (12) can map to 3-dimensional Schwarzschild like spacetime locally, but not globally.

It is well-known that the electric field is associated with the time component of the vector potential, $A_{t}$, while the magnetic field is associated with the angular component $A_{\varphi}$. Since we want to investigate the magnetic solutions, so we assume the vector potential as

$A_{\mu}=\left(\int F_{\varphi \rho} \mathrm{d} \rho\right) \delta_{\mu}^{\varphi}$.

Now we continue our paper for obtaining the magnetic solutions in the Einstein gravity and in presence of various models of NED.

\subsection{Static solutions}

\subsubsection{Class I: PMI NED model}

In this case, we want to obtain the solutions in presence of PMI NED and investigate the properties of the solutions. Therefore, we consider the PMI Lagrangian with the following form

$\mathcal{L}_{\mathrm{PMI}}(\mathcal{F})=(-\kappa \mathcal{F})^{s}$,

where $\kappa$ and $s$ are coupling and arbitrary constants, respectively. It is straightforward to show that for $s=1$, the PMI Lagrangian (14) reduces to the standard Maxwell Lagrangian $\left(\mathcal{L}_{\text {Maxwell }}(\mathcal{F})=-\kappa \mathcal{F}\right)$. Since the Maxwell invariant is negative, hereafter we set $\kappa=1$, without loss of generality. Using the nonlinear Maxwell equation (3) and the Lagrangian of PMI (14) with the metric (12), one can obtain

$F_{\varphi \rho}+K(\rho)=0$,

where

$K(\rho)=(2 s-1) \rho F_{\varphi \rho}^{\prime}$,

where the "prime" denotes differentiation with respect to $\rho$. Eq. (15) has the following solution

$F_{\varphi \rho}=\frac{q}{\rho^{1 /(2 s-1)}}$, where $q$ is an integration constant. In order to have physical asymptotical behavior, we restrict ourselves to $s>1 / 2$. To find the metric function $g(\rho)$, one may insert Eqs. (17) and (12) in the field equation (2). After some calculations, one can show that

$\left\{\begin{array}{ll}g^{\prime}(\rho)+2 \Lambda \rho-(2 s-1)\left(\frac{2 q^{2}}{l^{2} \rho^{1 /(2 s-1)}}\right)^{s}=0, & \rho \rho(\varphi \varphi) \text { component } \\ g^{\prime \prime}(\rho)+2 \Lambda+\left(\frac{2 q^{2}}{l^{2} \rho^{2 /(2 s-1)}}\right)^{s}=0, & t t \text { component }\end{array}\right.$,

It is straightforward to show that these equations have the following solutions

$g(\rho)=m-\Lambda \rho^{2}+\left\{\begin{array}{ll}\frac{2 q^{2} \ln \left(\frac{\rho}{l}\right)}{l^{2}}, & s=1 \\ \frac{2^{s-1}(2 s-1)^{2}}{(s-1)}\left(\frac{q}{l}\right)^{2 s} \rho^{2(s-1) /(2 s-1)}, & \text { otherwise }\end{array}\right.$,

where $m$ is the integration constant which is related to the mass parameter.

\subsubsection{Class II: exponential form of NED (ENED)}

Here, we consider ENED Lagrangian as

$\mathcal{L}_{\exp }(\mathcal{F})=\beta^{2}\left(\exp \left(-\frac{\mathcal{F}}{\beta^{2}}\right)-1\right)$,

where $\beta$ is the ENED parameter and in the limit $\beta \longrightarrow \infty$, $\mathcal{L}_{\text {exp }}(\mathcal{F})$ reduces to the standard Maxwell form $\mathcal{L}_{\text {Maxwell }}(\mathcal{F})=-\mathcal{F}$. Inserting the Lagrangian of ENED (20) in the nonlinear Maxwell equation (3) and using the metric (12), one can obtain

$\left[1-\left(\frac{2 F_{\varphi \rho}}{l \beta}\right)^{2}\right] F_{\varphi \rho}^{\prime}+\frac{F_{\varphi \rho}}{\rho}=0$.

This equation has the following solution

$F_{\varphi \rho}=\frac{l \beta}{2} \sqrt{-L_{W}}$

where $L_{W}=$ Lambert $W\left(-4 q^{2} / l^{2} \beta^{2} \rho^{2}\right)$ and the parameter $q$ is an integration constant. It is worthwhile to note that in order to have a real electromagnetic field, we should consider $\rho$ with the following limitation

$\rho>\rho_{0}=\frac{2 q}{l \beta} \exp \left(\frac{1}{2}\right)$.

Now, we want to obtain the function of $f(\rho)$. For this purpose, we can take into account Eqs. (12) and (22) in the 
gravitational field equation (2) to obtain its nonzero components as

$\left\{\begin{array}{lc}g^{\prime}(\rho)+\rho\left(2 \Lambda+\beta^{2}-\frac{2 \beta q}{l \rho}\left[\left(-L_{W}\right)^{-1 / 2}-\left(-L_{W}\right)^{1 / 2}\right]\right)=0, & \rho \rho(\varphi \varphi) \text { component } \\ g^{\prime \prime}(\rho)+\left(2 \Lambda+\beta^{2}-\frac{2 \beta q}{l \rho}\left(-L_{W}\right)^{-1 / 2}\right)=0, & t t \text { component }\end{array}\right.$,

After some calculations, one can show that these equations have the following solution

$g(\rho)=m-\Lambda \rho^{2}-\frac{\beta^{2} \rho^{2}}{2}+\int \frac{2 \beta q}{l}\left(\sqrt{-L_{W}}+\frac{1}{\sqrt{-L_{W}}}\right) \mathrm{d} \rho$.

\subsubsection{Class III: logarithmic form of NED (LNED)}

Now, we want to consider the LNED Lagrangian with the following form

$\mathcal{L}_{\log }(\mathcal{F})=-8 \beta^{2} \ln \left(1+\frac{\mathcal{F}}{8 \beta^{2}}\right)$

where $\beta$ is the LNED parameter and for weak nonlinearity limit $\beta \longrightarrow \infty, \mathcal{L}_{\log }(\mathcal{F})$ reduces to the standard Maxwell form $\mathcal{L}_{\text {Maxwell }}(\mathcal{F})=-\mathcal{F}$. Using the LNED Lagrangian (26) and the nonlinear Maxwell equation (3) with the metric (12), leads to

$\left[1-\left(\frac{F_{\varphi \rho}}{l \beta}\right)^{2}\right] F_{\varphi \rho}^{\prime}+\left[1+\left(\frac{F_{\varphi \rho}}{l \beta}\right)^{2}\right] \frac{F_{\varphi \rho}}{\rho}=0$,

with the following solution

$F_{\varphi \rho}=\frac{\rho l^{2} \beta^{2}}{2 q}(1-\Gamma)$,

where $\Gamma=\sqrt{1-(2 q / \rho l \beta)^{2}}$. In order to have a real electromagnetic field, we should consider $\rho$ with the following restriction

$\rho>\rho_{0}=\frac{2 q}{l \beta}$

Here, we want to obtain the solutions of Eqs. (2) and (3). Considering Eqs. (12) and (26), one can obtain the nonzero components as

$\left\{\begin{array}{ll}g^{\prime \prime}(\rho)+2 \Lambda+8 \Pi \beta^{2}=0, & t t \text { component } \\ {\left[g^{\prime}(\rho)+2 \Lambda \rho+8 \rho \beta^{2}(\Pi-2)\right](\Gamma-1)+\frac{32 q^{2}}{\rho l^{2}}=0,} & \rho \rho(\varphi \varphi) \text { component }\end{array}\right.$, where

$$
\Pi=\ln \left(\frac{l^{2} \rho^{2} \beta^{2}(1-\Gamma)}{2 q^{2}}\right) .
$$

After some calculations, one can determine the metric function $g(\rho)$ as

$$
\begin{aligned}
g(\rho)= & m-\Lambda \rho^{2}+\frac{8 q^{2}}{l^{2}} \ln [l \rho \beta(1+\Gamma)] \\
& -2 \rho^{2} \beta^{2}[3(1-\Gamma)+2 \Pi] .
\end{aligned}
$$

\subsection{Energy condition}

In order to find a physical solutions, we examine the energy conditions for these nonlinear models. It is usual to consider the orthonormal contravariant basis vectors and calculate the three dimensional energy momentum tensor as $T^{\mu \nu}=\operatorname{diag}\left(\mu, p_{r}, p_{t}\right)$. The physical concepts of $\mu, p_{r}$ and $p_{t}$ are, respectively, the energy density, the radial pressure and the tangential pressure. Having the energy momentum tensor at hand, we are in a position to discuss energy conditions. We use the following known constraints in three dimensions (Table 1).

In order to simplify the mathematics and physical interpretations, we use the following orthonormal contravariant (hatted) basis vectors for diagonal static metric (12)

$\mathbf{e}_{\hat{t}}=\frac{l}{\rho} \frac{\partial}{\partial t}, \mathbf{e}_{\widehat{\rho}}=\sqrt{g} \frac{\partial}{\partial \rho}, \mathbf{e}_{\widehat{\phi}}=\frac{1}{l \sqrt{g}} \frac{\partial}{\partial \phi}$.

It is a matter of straightforward calculations to show that the nonzero components of stress-energy tensor for the mentioned models are

$$
T^{\hat{t}}= \begin{cases}\frac{1}{2}\left(\frac{2 F_{\phi \rho}^{2}}{l^{2}}\right)^{s}, & \text { PMI } \\ \frac{\beta^{2}}{2}\left[1-\exp \left(\frac{-2 F_{\phi \rho}^{2}}{l^{2} \beta^{2}}\right)\right], & \text { ENED } \\ 4 \beta^{2} \ln \left[1+\left(\frac{F_{\phi \rho}}{2 l \beta}\right)^{2}\right], & \text { LNED }\end{cases}
$$

Table 1 Energy conditions criteria

\begin{tabular}{ll}
\hline$p_{r}+\mu \geq 0$ & For null energy condition (NEC) \\
$p_{t}+\mu \geq 0$, & \\
$\mu \geq 0$ & For weak energy condition (WEC) \\
$p_{r}+\mu \geq 0$, & \\
$p_{t}+\mu \geq 0$ & \\
$\mu \geq 0$ & For dominant energy condition (DEC) \\
$-\mu \leq p_{r} \leq \mu$, & \\
$-\mu \leq p_{t} \leq \mu$ & \\
$p_{r}+\mu \geq 0$ & For strong energy condition (SEC) \\
$p_{t}+\mu \geq 0$ & \\
$\mu+p_{r}+p_{t} \geq 0$ & \\
\hline
\end{tabular}



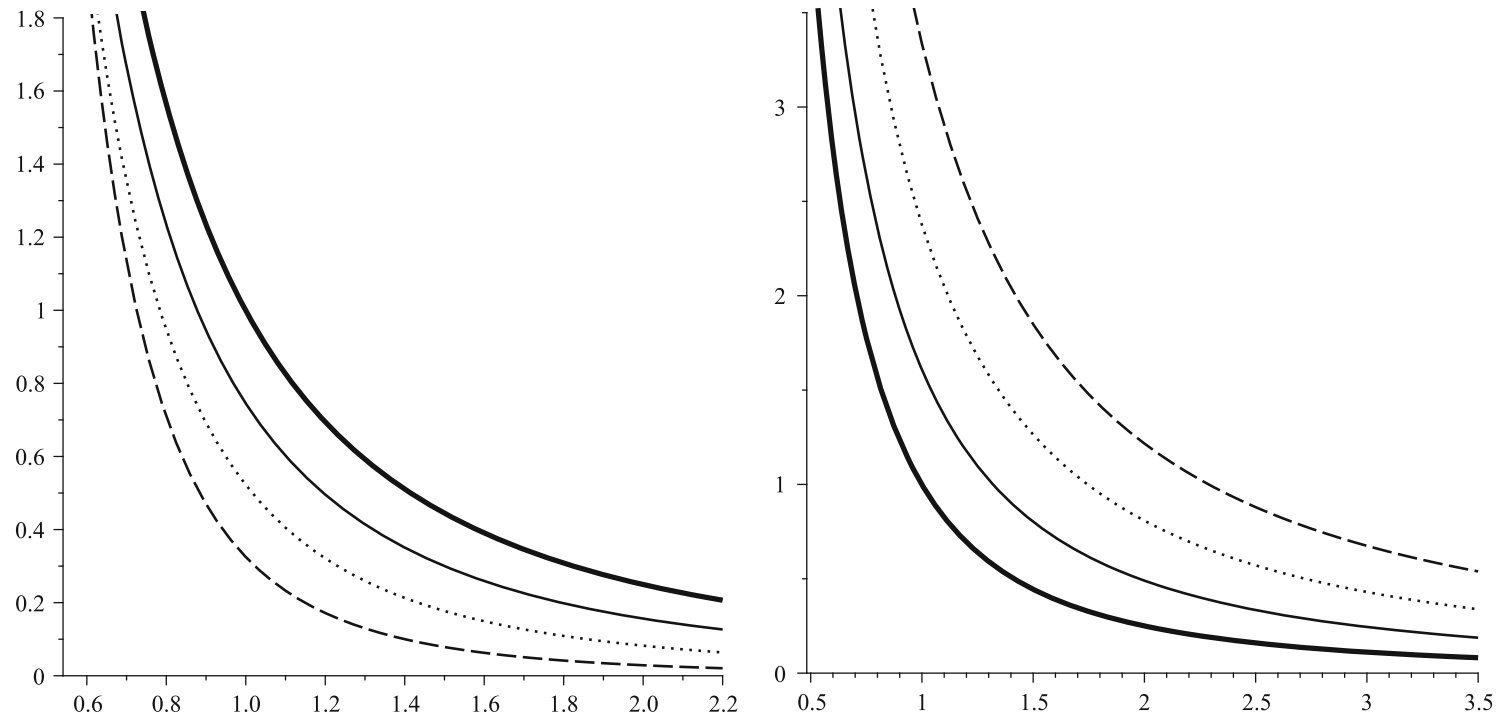

Fig. 1 Maxwell and PMI solutions: $T^{\widehat{\rho} \widehat{\rho}}$ versus $\rho$ for $l=1$ and $q=1$. Left diagram $s=0.7$ (dashed line), $s=0.8$ (dotted line), $s=0.9$ (continuous line) and $s=1$ (bold line). Right diagram $s=1$ (bold line), $s=1.2$ (continuous line), $s=1.4$ (dotted line) and $s=1.6($ dashed line)

$$
T^{\widehat{\rho} \widehat{\rho}}=T \widehat{\phi} \widehat{\phi}= \begin{cases}\frac{2 s-1}{2}\left(\frac{2 F_{\phi \rho}^{2}}{l^{2}}\right)^{s}, & P M I \\ -\frac{\beta^{2}}{2}\left[1-\left(1+\frac{4 F_{\phi \rho}^{2}}{l^{2} \beta^{2}}\right) \exp \left(\frac{-2 F_{\phi \rho}^{2}}{l^{2} \beta^{2}}\right)\right], & E N E D . \\ -4 \beta^{2}\left(\ln \left[1+\left(\frac{F_{\phi \rho}}{2 l \beta}\right)^{2}\right]-\frac{F_{\phi r}^{2}}{2 l^{2} \beta^{2}\left[1+\left(\frac{F_{\phi \rho}}{2 l \beta}\right)^{2}\right]}\right), & \text { LNED }\end{cases}
$$

After some calculations, one finds that NEC, WEC and SEC are satisfied for all models, simultaneously. In addition, it is easy to show that DEC is satisfy for both ENED and LNED branches, while for PMI case it can be satisfied for $\frac{1}{2}<s \leq 1$.

In order to investigate the effect of the nonlinearity of the models on the energy density of the spacetime, we plot the $T$ tit versus $\rho>\rho_{0}$ for different values of nonlinearity parameter (s or $\beta$ ).

As one can see, in case of PMI theory, we have a special case $s=1$ which is denoted as Maxwell theory of electrodynamics. We consider two set of values for $s$ in order to have a better understanding of the behavior of energy density. These two cases are $\frac{1}{2}<s<1$ and $s>1$. It is evident through studying these two cases (Fig. 1) that as one increases $s$ parameter, the concentration of energy density increases. In other words, for a fixed value of $\rho$ the lowest value of the energy density of spacetime belongs to the lowest value of $s$. In general, the energy density is a decreasing function of $\rho$.

Next for the two BI-types nonlinear electromagnetic fields we have plotted Fig. 2. Due to structure of their nonlinear electrodynamics, these theories do not have real valued energy tensor every where. There is a region in which their energy tensor is imaginary. This region is a decreasing function of nonlinearity parameter. As one can see in case of these two theories, increasing the nonlinearity parameter leads to decreasing the concentration of energy density. In other words, in order to decrease the concentration of energy density, one should increase the nonlinearity parameter, $\beta$.

Finally we have plotted Fig. 3 in order to make a comparison between these theories of electromagnetic fields. It is evident that the lowest and highest value of energy density belongs to PMI theory. Regardless of PMI case, the LNED has larger value of energy density and next one is ENED.

\subsection{Geometric properties}

Now we want to study the properties of the spacetime described by Eq. (12) with obtained metric functions of different NED models. First of all, to investigate the singularities and asymptotical behavior of the solutions, we calculate the Kretschmann scalar

$$
\begin{aligned}
& \left.R_{\mu \nu \lambda \kappa} R^{\mu \nu \lambda \kappa}\right|_{\mathrm{PMI}} \\
& =12 \Lambda^{2}-4(4 s-3) \Lambda \mathcal{D}+\left(8 s^{2}-8 s+3\right) \mathcal{D}^{2} \\
& \mathcal{D}=\left(\frac{\sqrt{2} q}{l \rho^{1 /(2 s-1)}}\right)^{2 s} \\
& \left.R_{\mu \nu \lambda \kappa} R^{\mu \nu \lambda \kappa}\right|_{\exp }=12 \Lambda^{2}+4 \Lambda(2 \mathcal{J}+\mathcal{K})-\left(2 \mathcal{J}^{2}+\mathcal{K}^{2}\right) \\
& \mathcal{J}=\beta^{2}-\frac{2 q \beta\left(L_{W}-1\right)}{\rho l \sqrt{-L_{W}}} \\
& \mathcal{K}=\beta^{2}+\frac{2 q \beta}{\rho l \sqrt{-L_{W}}} \\
& \left.R_{\mu \nu \lambda \kappa} R^{\mu \nu \lambda \kappa}\right|_{\log }=12 \Lambda^{2}-96 \Lambda \beta^{2}\left(\Pi+\frac{2}{3}(1-\Gamma)\right)+\mathcal{T}
\end{aligned}
$$




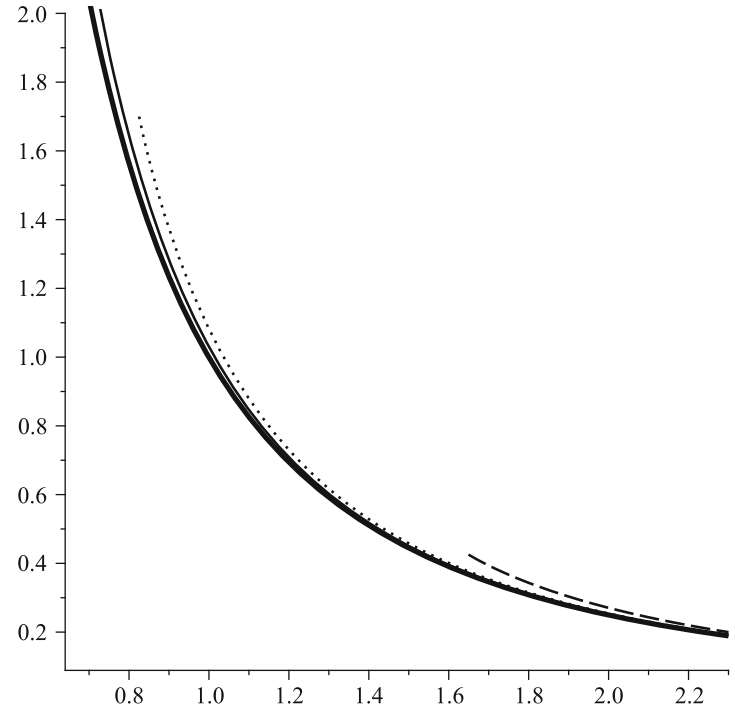

Fig. 2 ENED and LNED solutions: $T \widehat{\widehat{\rho} \widehat{\rho}}$ versus $\rho$ for $l=1$ and $q=1$. Left diagram (ENED) $\beta=2$ (dashed line), $\beta=4$ (dotted line), $\beta=6$ (continuous line) and Maxwell case (bold line). Right diagram (LNED):

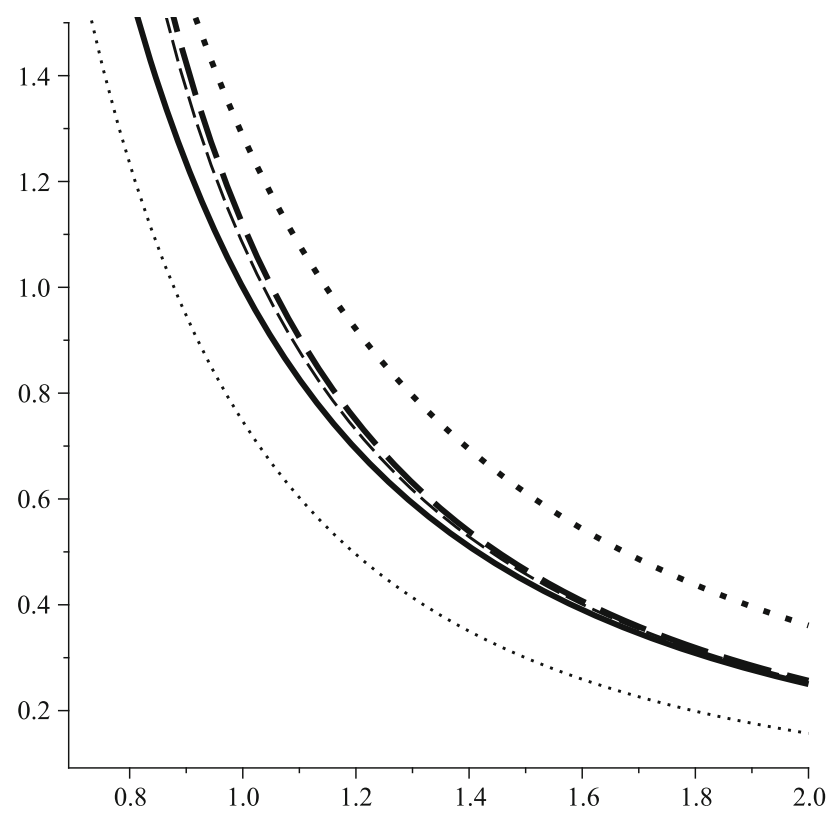

Fig. 3 Comparison of various theories: $T^{\widehat{\rho} \widehat{\rho}}$ versus $\rho$ for $l=1, q=1$, $s=0.9$ (dotted line for PMI), $s=1$ (bold continuous line for Maxwell), $s=1.1$ (bold dotted line for PMI), $\beta=4$ (dashed line for ENED) and $\beta=4$ (bold dashed line for LNED)

$$
\begin{aligned}
\mathcal{T}= & 192 \beta^{2}\left\{\left(\ln \left[2 q^{2} l^{2} \rho^{2} \beta^{2}(1-\Gamma)\right]\right)^{2}\right. \\
& -\frac{4}{3}(1-\Gamma)\left[1-\ln \left[l^{2} \rho^{2} \beta^{2}(1-\Gamma)\right]\right] \\
& \left.+\frac{4}{3}\left(1-\frac{1}{l \rho \beta}\right) \ln \left(2 q^{2}\right)-\frac{8 q^{3}}{3 l^{2} \rho^{2} \beta^{2}}\right\} .
\end{aligned}
$$

Regarding Eqs. (34), (36) and (39), it is easy to show that Kretschmann scalar diverges at $\rho=0$ and therefore one

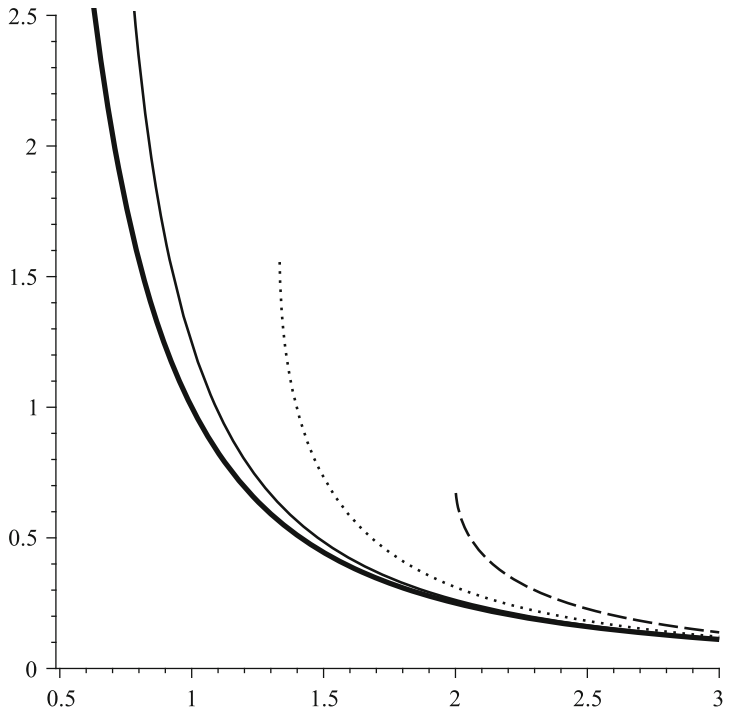

$\beta=1$ (dashed line), $\beta=1.5$ (dotted line), $\beta=3$ (continuous line) and Maxwell case (bold line)

might think that there is a curvature singularity located at $\rho=0$; but as we will see, the spacetime will never achieve $\rho=0$. Also, in the mentioned equations, the Kretschmann scalar reduces to $12 \Lambda^{2}$ for $r \longrightarrow \infty$, which confirms asymptotical behavior of these spacetimes is AdS. There are two possible cases for the metric function. In one case, the metric function has no root which is interpreted as naked singularity. In the other case, metric function has one or more than one root. If one consider $r_{0}$ as the largest root of metric function, there will be a change in signature of metric. In other words, for $\rho<r_{0}$ metric function is negative, hence metric signature is $(-,+,+)$. For $\rho>r_{0}$ metric function is positive, therefore metric signature is $(-,-,-)$. This change in metric signature results into a conclusion: it is not possible to extend spacetime to $\rho<r_{0}$.

In order to cover the whole spacetime, correctly, we introduce another coordinate transformation. The new radial coordinate $r$ may be introduces as

$r^{2}=\rho^{2}-r_{0}^{2} \Longrightarrow \mathrm{d} \rho^{2}=\frac{r^{2}}{r^{2}+r_{0}^{2}} \mathrm{~d} r^{2}$,

where $\rho \geq r_{0}$ leads to $r \geq 0$. Applying this coordinate transformation, the metric (12) should be written as

$\mathrm{d} s^{2}=-\frac{r^{2}+r_{0}^{2}}{l^{2}} \mathrm{~d} t^{2}+\frac{r^{2}}{\left(r^{2}+r_{0}^{2}\right) g(r)} \mathrm{d} r^{2}+l^{2} g(r) \mathrm{d} \varphi^{2}$,

where the coordinates $r$ assumes the values $0 \leq r<\infty$, and obtained $g(r)$ 's (Eqs. (19), (25) and (32)), are now given as 


$$
\begin{aligned}
& \left.g(r)\right|_{\mathrm{PMI}}=m-\Lambda\left(r^{2}+r_{0}^{2}\right) \\
& \quad+ \begin{cases}\frac{q^{2} \ln \left(\frac{r^{2}+r_{0}^{2}}{l^{2}}\right)}{l^{2}}, & s=1 \\
\frac{2^{s-1}(2 s-1)^{2}}{(s-1)}\left(\frac{q}{l}\right)^{2 s}\left(r^{2}+r_{0}^{2}\right)^{(s-1) /(2 s-1)}, & \text { otherwise }\end{cases}
\end{aligned}
$$

$$
\begin{aligned}
\left.g(r)\right|_{\exp }= & m-\Lambda\left(r^{2}+r_{0}^{2}\right)-\frac{\beta^{2}\left(r^{2}+r_{0}^{2}\right)}{2} \\
& +\int \frac{4 \beta q r}{l \sqrt{r^{2}+r_{0}^{2}}}\left(\sqrt{-L_{W}^{\prime}}+\frac{1}{\sqrt{-L_{W}^{\prime}}}\right) \mathrm{d} r
\end{aligned}
$$

$L_{W}^{\prime}=$ Lambert $W\left(-\frac{4 q^{2}}{l^{2} \beta^{2}\left(r^{2}+r_{0}^{2}\right)}\right)$,

$$
\left.g(r)\right|_{\log }=m-\Lambda\left(r^{2}+r_{0}^{2}\right)+\frac{8 q^{2}}{l^{2}} \ln \left[l \beta(1+\Gamma) \sqrt{r^{2}+r_{0}^{2}}\right]
$$$$
-2\left(r^{2}+r_{0}^{2}\right) \beta^{2}[3(1-\Gamma)+2 \Pi],
$$

$$
\begin{aligned}
\Gamma^{\prime} & =\sqrt{1-\left(\frac{2 q}{l \beta \sqrt{r^{2}+r_{0}^{2}}}\right)^{2}}, \\
\Pi^{\prime} & =\ln \left(\frac{l^{2} \beta^{2}\left(r^{2}+r_{0}^{2}\right)\left(1-\Gamma^{\prime}\right)}{2 q^{2}}\right) .
\end{aligned}
$$

The nonzero component of electromagnetic field in the new coordinate can be given by

$$
\begin{aligned}
& \left.F_{\varphi r}\right|_{\mathrm{PMI}}=q\left(r^{2}+r_{0}^{2}\right)^{-1 /(4 s-2)}, \\
& \left.F_{\varphi r}\right|_{\exp }=\frac{l \beta}{2} \sqrt{-L_{W}^{\prime}}, \\
& \left.F_{\varphi r}\right|_{\log }=\frac{l^{2} \beta^{2}}{2 q} \sqrt{r^{2}+r_{0}^{2}}\left(1-\Gamma^{\prime}\right) .
\end{aligned}
$$

One can show that all curvature invariants do not diverge in the range $0 \leq r<\infty$ and also $g(r)$, in different NED models namely Eqs. (43), (44) and (46), is positive definite for $0 \leq r<\infty$. It is evident through studying the obtained values that in order to solutions contain singularity both $r$ and $r_{0}$ must be zero whereas this case is never reached due to considering nonzero value for $r_{0}$. Therefore, these spacetimes have no curvature singularity and horizon. However, the spacetime (42) has a conic geometry because the limit of the ratio "circumference/radius" is not $2 \pi$ and therefore the spacetime has a conical singularity at $r=0$

$\lim _{r \rightarrow 0} \frac{1}{r} \sqrt{\frac{g_{\varphi \varphi}}{g_{r r}}} \neq 1$
The conical singularity can be removed if one exchanges the coordinate $\varphi$ with the following period

$\operatorname{Period}_{\varphi}=2 \pi\left(\lim _{r \rightarrow 0} \frac{1}{r} \sqrt{\frac{g_{\varphi \varphi}}{g_{r r}}}\right)^{-1}=2 \pi(1-4 \mu)$

where $\mu$ is given by

$\mu=\frac{1}{4}+\frac{1}{\Omega}$

where $\Omega$ is different for various models of NED. We find that

$\left.\Omega\right|_{\mathrm{PMI}}=4 l r_{0}\left[\Lambda-\frac{(2 s-1)}{2}\left(\frac{\sqrt{2} q}{l r_{0}^{1 /(2 s-1)}}\right)^{2 s}\right]$,

where for $s=1$ this equation reduces to the Maxwell theory. In order to have better understanding of the behavior of deficit angle, we calculate the divergence points of the deficit angle in PMI model in which these points are located at

$\left.r_{0}\right|_{\delta \varphi \rightarrow \infty}= \pm\left[\frac{(2 s-1)}{2 \Lambda}\left(\frac{\sqrt{2} q}{l}\right)^{2 s}\right]^{\frac{2 s-1}{2 s}}$

Due to complexity of obtained relation for deficit angle, it is not possible to calculate roots of deficit angle analytically. Therefore we will study them in context of graphs for deficit angle later. It is worthwhile to mention that in case of Maxwell theory the divergency and roots of deficit angle are obtained as follow

$\left.r_{0}\right|_{\delta \varphi \rightarrow \infty}= \pm \frac{q}{l \sqrt{\Lambda}}$

$\left.r_{0}\right|_{\delta \varphi=0}=\frac{ \pm \sqrt{1+4 \Lambda q^{2}}-1}{2 l \Lambda}$.

As one can see, in case of Maxwell theory, the divergency is only seen in dS spacetime. In other words, in AdS spacetime which is of our interest in this paper, no divergency exists. In case of PMI theory, one can draw same conclusion; no divergency is observed in AdS spacetime.

Next, using the method that was introduced for calculation of deficit angle, in case of ENED, we have the following result

$$
=\frac{q \mathcal{N} \mathcal{E}\left(2+\frac{q \mathcal{E} \sqrt{-L_{\mathcal{W}}}}{l \beta r_{0}}\right)+8 q \beta^{2} l^{2} \sqrt{-L_{\mathcal{W}}}\left(1+L_{\mathcal{W}}\right)+\frac{l \beta r_{0} \mathcal{N}}{\sqrt{-L_{\mathcal{W}}}}}{\frac{\beta l^{2} r_{0}^{2}\left(1+L_{\mathcal{W}}\right)}{8}\left(2+\frac{2 q \mathcal{E} \sqrt{-L_{\mathcal{W}}}}{l \beta r_{0}}\right)^{2}},
$$

where

$L_{\mathcal{W}}=$ Lambert $W\left(-\frac{4 q^{2}}{l^{2} \beta^{2} r_{0}^{2}}\right)$, 

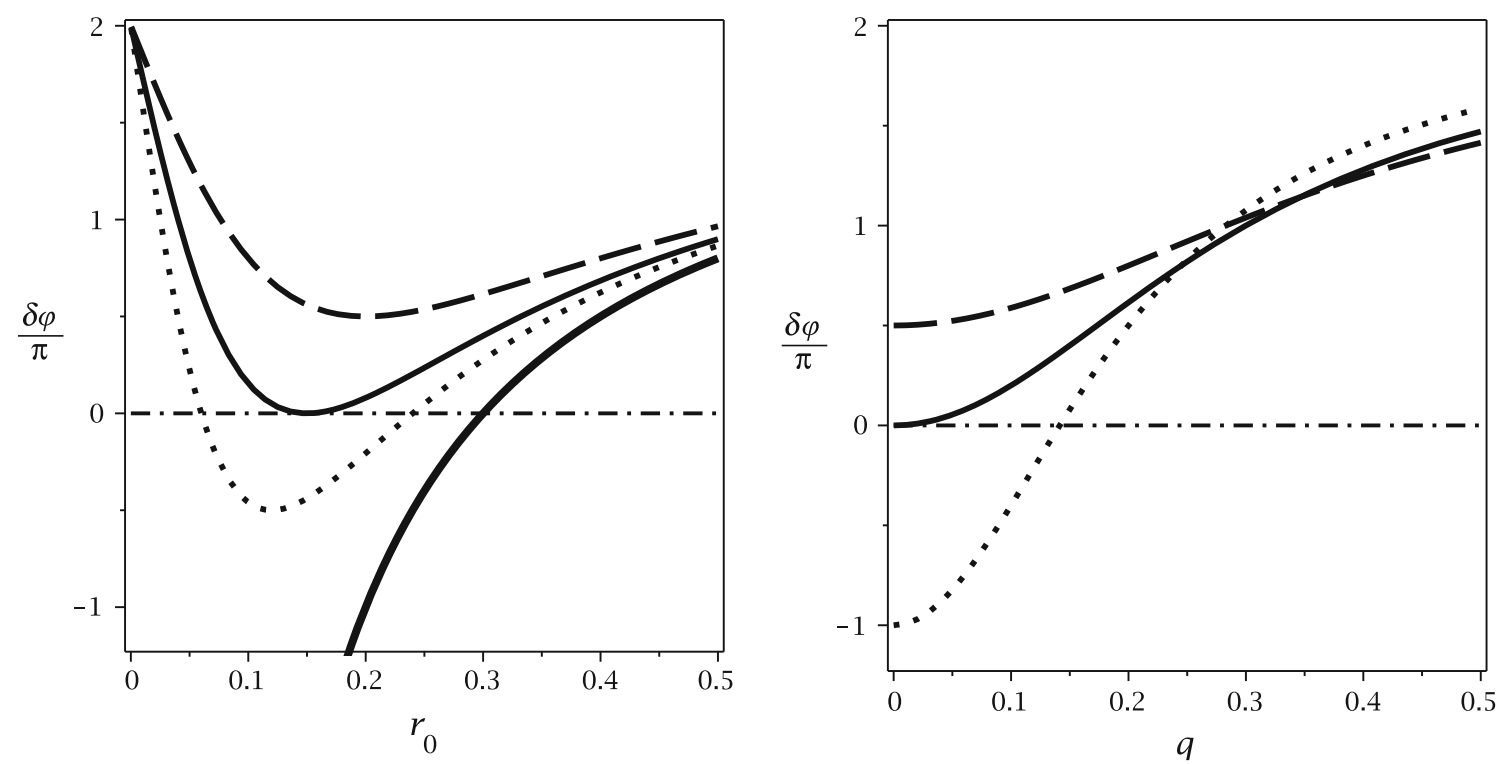

Fig. 4 Maxwell solutions: $\delta \phi / \pi$ versus $r_{0}$ (left) and $\delta \phi / \pi$ versus $q$ (right) for $l=0.3$. Left diagram $q=0$ (bold line), $q=0.12$ (dotted line), $q=0.15$ (continuous line) and $q=0.2$ (dashed line). Right diagram $r_{0}=0.2($ dotted line $), r_{0}=0.3$ (continuous line) and $r_{0}=0.4($ dashed line)

$$
\begin{aligned}
\mathcal{N}= & 2 q l \beta r_{0} L_{\mathcal{W}}^{2}+\sqrt{-L_{\mathcal{W}}} \\
& \times\left[l^{2} r_{0}^{2}\left(1+L_{\mathcal{W}}\right)\left(2 \Lambda+\beta^{2}\right)+4 q^{2} \exp \left(-\frac{1}{2} L_{\mathcal{W}}\right)\right],
\end{aligned}
$$

$\mathcal{E}=E i\left(1, \frac{1}{2} L_{\mathcal{W}}\right)$

and the exponential integral, $E i(a, z)$, are defined for $\operatorname{Re}(z)>0(\operatorname{Re}(z)$ means the real part of $z)$ by

$E i(a, z)=\int_{1}^{\infty} \exp (-z x) x^{-a} \mathrm{~d} x$.

It is notable to mention that, in order to have a real deficit angle, $\delta \phi$, we should consider $\beta>\beta_{\min }$, where

$\beta_{\min }=\frac{2 q}{l r_{0}} \exp \left(\frac{1}{2}\right)$

By applying same process for the case of LNED, one can find

$\left.\Omega\right|_{\log }=16 \beta^{2} \operatorname{lr} 0\left\{1+\frac{\Lambda}{4 \beta^{2}}-\mathcal{H}+\ln \left[-\frac{2}{\beta(1-\mathcal{H})}\left(\frac{q l}{r_{0}}\right)^{2}\right]\right\}$,

$\mathcal{H}=\sqrt{1+\left(\frac{2 q l}{\beta r_{0}}\right)^{2}}$

For logarithmic and exponential forms, due to complexity of obtained deficit angle relations, it is not possible to find singular points of deficit angle, analytically. But by employing numerical method, it was seen that the singularity is located at $r_{0}=0$, which we should note that, the system never reaches this limit.

Now, we are in position to study the effects of variation of different parameters on the deficit angle in these nonlinear theories. To do so, we have plotted Figs. 4, 5, 6, 7 and 8.

In case of PMI theory, obtained results are as follow. Due to structure of equation of deficit angle in the presence of this nonlinear electromagnetic field, small values of $s(0<s<1 / 2)$ are considered non physical. Therefore, we will only consider large values of $s(s>1 / 2)$. As for the case $s=1$, the PMI theory will reduce to Maxwell theory, before we study nonlinear theories, we first investigate the properties of the Maxwell solutions.

To investigate the effects of charge on the deficit angle, we plot Fig. 4 (left). In this case, there is a minimum for deficit angle located at $r_{0_{\min }}$. This $r_{0_{\min }}$ and corresponding deficit angle to it are increasing functions of charge. In the other words, there is a critical value of charge, $q_{c}$, which for $q<q_{c}$, deficit angle has two roots and a region of negativity whereas for $q>q_{c}$, deficit angle has no root and always positive. It is worthwhile to mention that in absence of charge, deficit angle is only an increasing function of $r_{0}$.

In case of varying $r_{0}$ (Fig. 4 right), deficit angle is an increasing function of charge. In this case, there also exists a critical value, $r_{0_{c}}$ in which for $r_{0}<r_{0_{c}}$, there will be a region of negativity and a root for calculated values of deficit angle. For case of $r_{0}>r_{0_{c}}$, deficit angle is positive and without any root. It is worthwhile to mention that for small values of charge the highest values of deficit angle belongs to the highest value of $r_{0}$. As charge increases large enough, this 

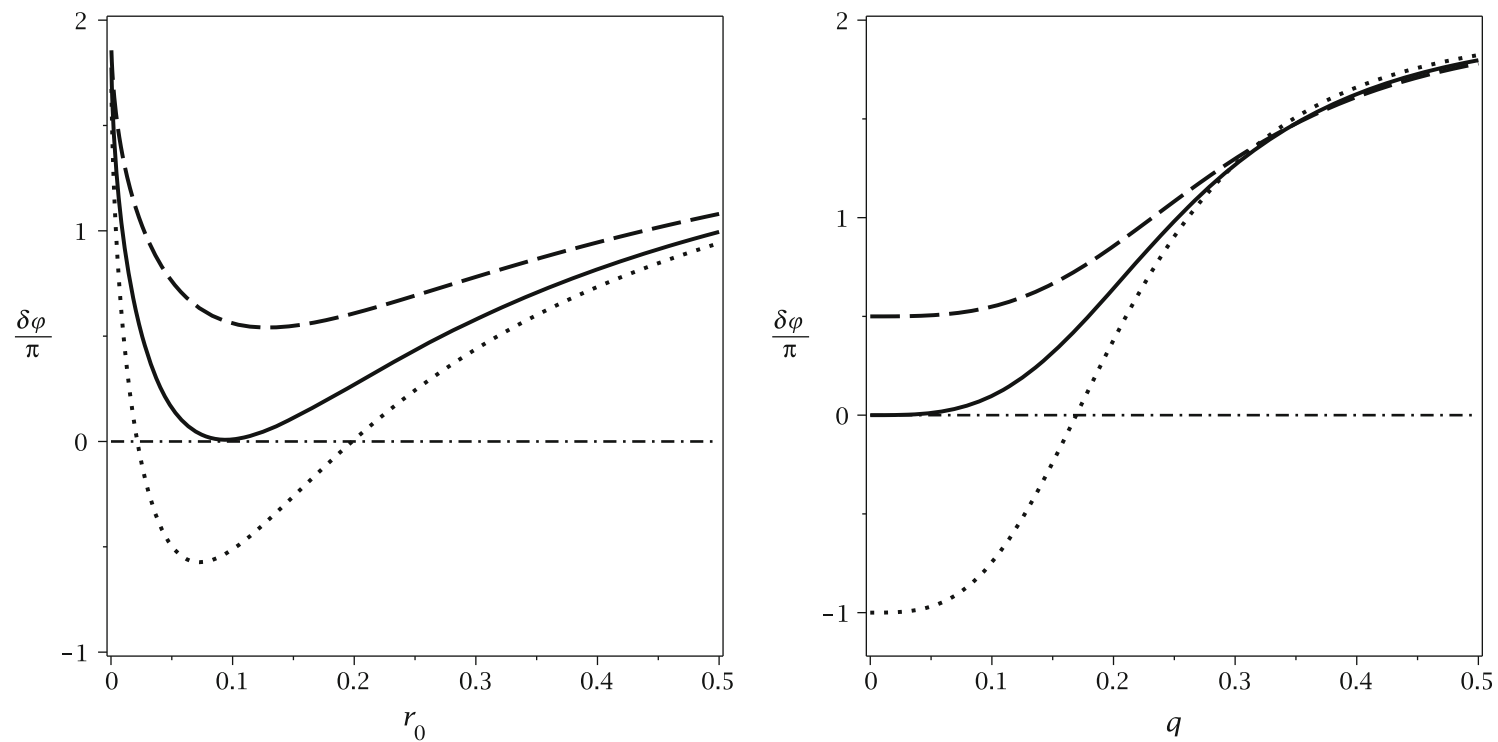

Fig. 5 PMI solutions: $\delta \phi / \pi$ versus $r_{0}$ (left) and $\delta \phi / \pi$ versus $q$ (right) for $l=0.3$ and $s=1.6$. Left diagram $q=0.17$ (dotted line), $q=0.191$ (continuous line) and $q=0.22$ (dashed line). Right diagram $r_{0}=0.2($ dotted line $), r_{0}=0.3$ (continuous line) and $r_{0}=0.4$ (dashed line)
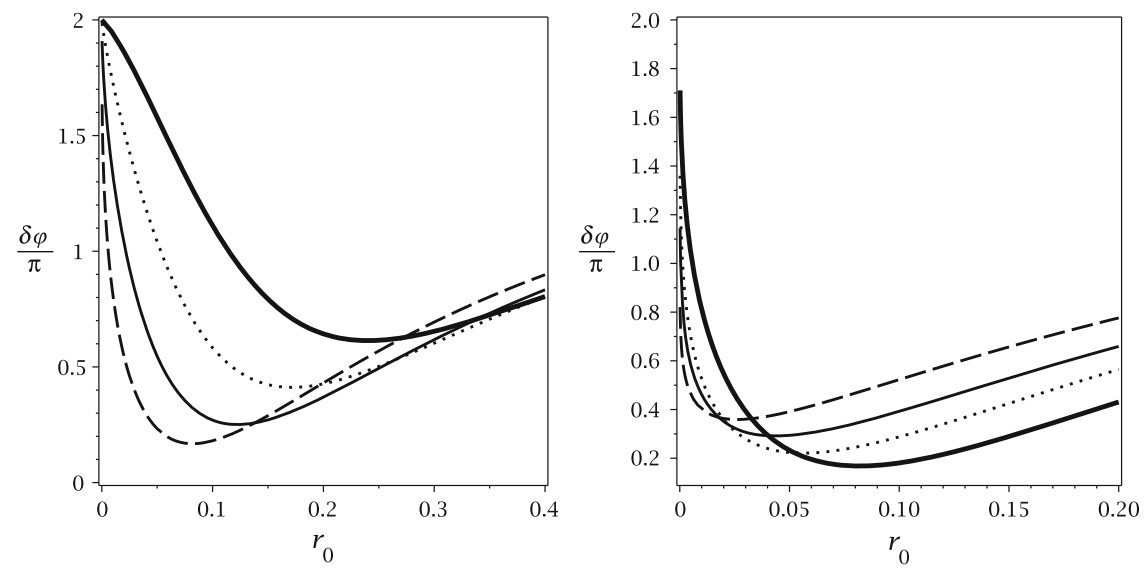

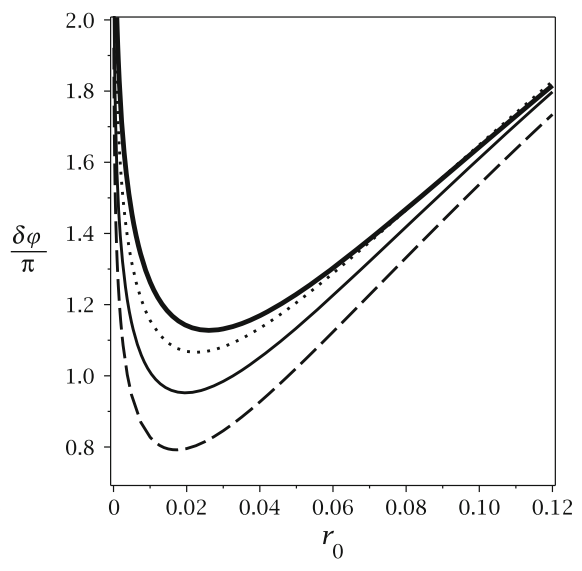

Fig. 6 PMI solutions $\delta \phi / \pi$ versus $r_{0}$ for $l=0.3$ and $q=0.2$. Left diagram $s=0.9$ (bold line), $s=1.1$ (dotted line), $s=1.4$ (continuous line) and $s=2$ (dashed line). Middle diagram $s=2$ (bold line), $s=3$ (dotted line), $s=4$ (continuous line) and $s=7$ (dashed line). Right diagram $s=7$ (bold line), $s=8$ (dotted line), $s=9$ (continuous line) and $s=10$ (dashed line) behavior will change; the highest values of the deficit angle belongs to the lowest value of $r_{0}$.

As in case of PMI theory for variation of charge Fig. 5 (left) is plotted. For this case, there is a minimum $r_{0_{\min }}$ and the deficit angle corresponding to $r_{0}=r_{0_{\min }}$ are increasing functions of charge. There is a critical value for charge, $q_{c}$ in which deficit angle corresponds to it, is zero. For $q<q_{c}$, there will be two roots for deficit angle, otherwise deficit angle does not have root. Next, in order to study the effects of variation of $r_{0}$, we have Fig. 5 (right). The behavior of deficit angle and the effects of varying $r_{0}$ is similar to the case of varying $r_{0}$ in the Maxwell theory.

As for the effects of $s$, we plot Fig. 6. Interestingly, different behaviors are seen for different values of $s$. The general behavior of the graphs are similar to variation of charge. But with one unique property. There is a different behavior for plotted graphs of deficit angle. First the $r_{0_{\min }}$ and corresponding deficit angle to it are decreasing functions of $s$ (Fig. 6 left). This behavior will change as one increases $s$ which results into $r_{0_{\min }}$ being a decreasing function of $s$ whereas deficit angle corresponding to $r_{0}=r_{0_{\min }}$ is an increasing function of $s$ (Fig. 6 middle). This behavior will change again if one increases $s$ which leads to $r_{0_{\min }}$ and deficit angle of it being a decreasing function of $s$ (Fig. 6 right).

As for exponential form of nonlinear electromagnetic field, it is seen that due to its structure, there is a divergency for deficit angle for the cases of the deficit angle versus nonlinearity parameter (Fig. 7 left and middle). It is evident that 

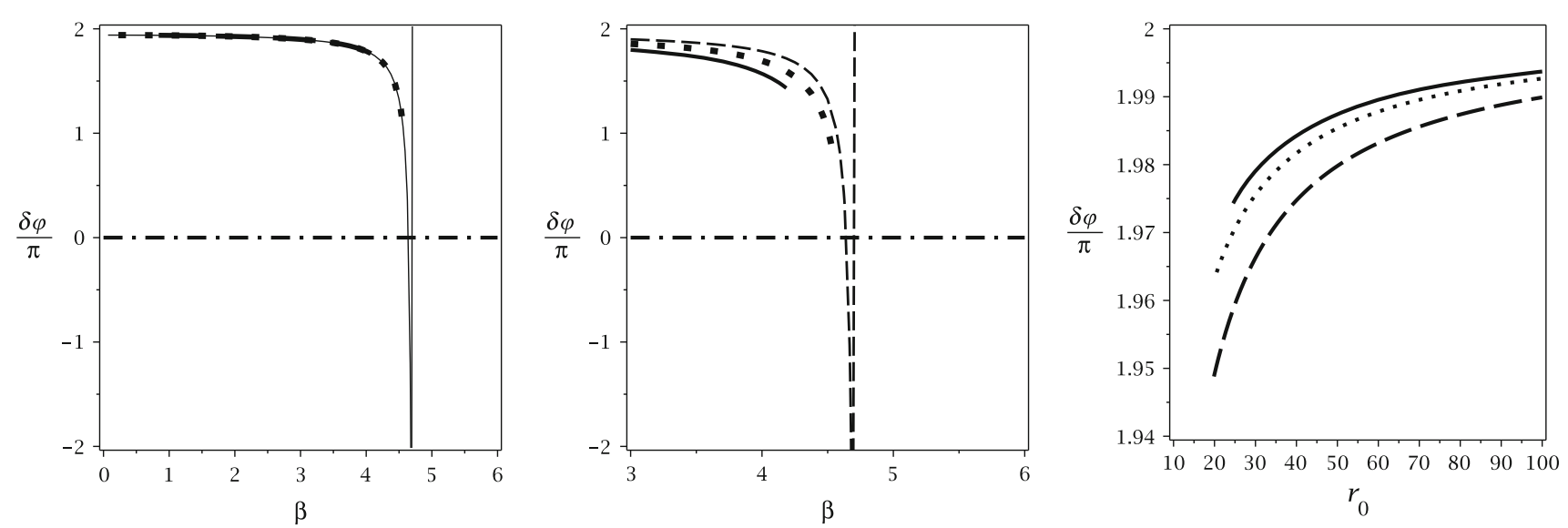

Fig. 7 ENED solutions: $\delta \phi / \pi$ versus $\beta$ (left and middle) and $\delta \phi / \pi$ versus $r_{0}$ (right) for $l=0.3$ Left diagram $r_{0}=10, q=0.06$ (continuous line), $q=0.1$ (dotted line) and $q=0.2$ (dashed line). Middle diagram

$q=0.06, r_{0}=5$ (continuous line $), r_{0}=7$ (dotted line) and $r_{0}=10$ (dashed line). Right diagram $q=1, \beta=1$ (continuous line), $\beta=2$ (dotted line) and $\beta=3$ (dashed line)
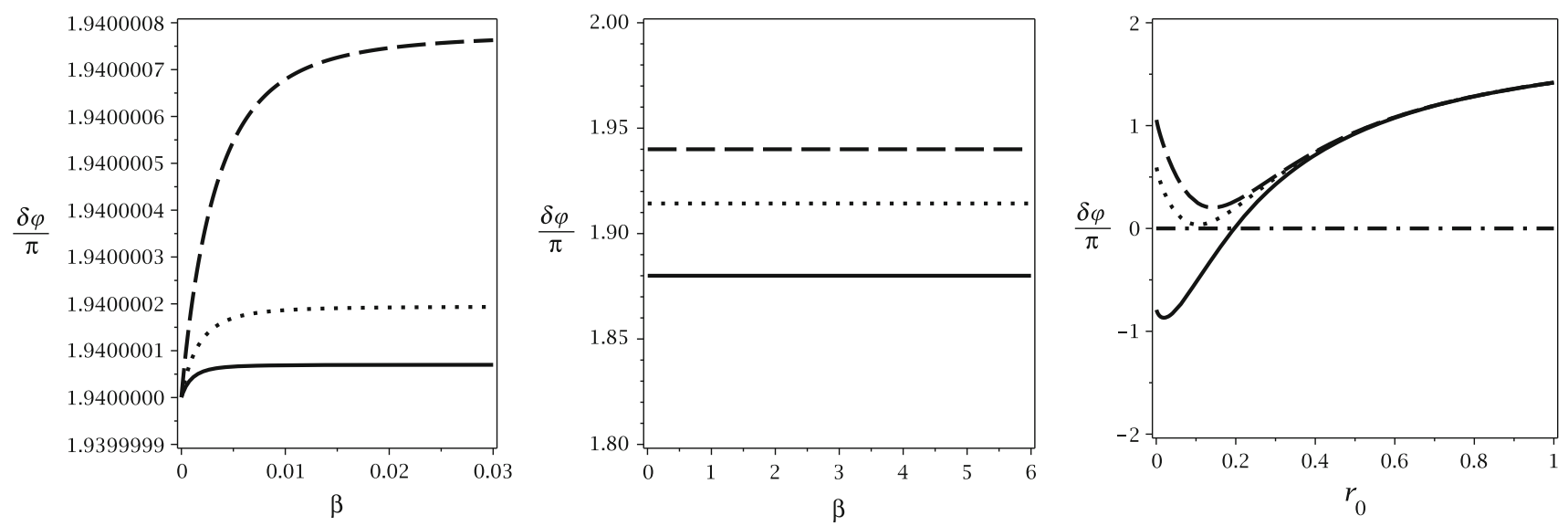

Fig. 8 LNED solutions: $\delta \phi / \pi$ versus $\beta$ (left and middle) and $\delta \phi / \pi$ versus $r_{0}$ (right) for $l=0.3$ Left diagram $r_{0}=10, q=0.06$ (continuous line), $q=0.1$ (dotted line) and $q=0.2$ (dashed line). Middle diagram

$q=0.06, r_{0}=5$ (continuous line), $r_{0}=7$ (dotted line) and $r_{0}=10$ (dashed line). Right diagram $q=1, \beta=1$ (continuous line), $\beta=2$ (dotted line) and $\beta=3$ (dashed line)

the existence of the divergency is a function of the variations of charge and $r_{0}$. In other words, for sufficiently small (large) values of charge $\left(r_{0}\right)$, there will be a divergency for deficit angle. Whereas, by increasing (decreasing) charge $\left(r_{0}\right)$ instead of divergency, there will be a region in which deficit angle is not real. This region is an increasing (decreasing) function of the charge $\left(r_{0}\right)$ (Fig. 7 left and middle).

Next, for the effects of nonlinearity parameter on deficit angle (Fig. 7 right), the region where deficit angle is not real is seen in this case too. It is evident that this region is a decreasing function of nonlinearity parameter. Overall, the deficit angle in this case is an increasing function of the $r_{0}$ whereas for the case of the nonlinearity, it is a decreasing function of the $\beta$. This shows that effects of the these two parameters on deficit angle are opposite of each other.

For the case of logarithmic nonlinear electromagnetic field, one can find following results. As for the effects of charge, plotted graph (Fig. 8 left) shows that deficit angle is an increasing function of nonlinearity parameter and for case of fixing nonlinearity and other parameters, the highest value of deficit angle belongs to the highest value of charge. On the other hand, as for the effects of $r_{0}$ (Fig. 8 middle and Fig. 9), the calculated values of deficit angle are positive and for $\beta=0$ deficit angle is also positive and non zero. As $r_{0}$ decreases, the related value of deficit angle for case of $\beta=0$, decreases. In general, in this case too, the deficit angle is an increasing function of the nonlinearity parameter. In order to show this behavior, we have plotted Fig. 9.

For the effects of nonlinearity parameter, Fig. 8 (right) is plotted. As one can see, interestingly, the behavior of this graph is quite different comparing to previous case. Deficit angle is positive and non zero for case of $r_{0}=0$. The value of deficit angle for this case is an increasing function of nonlinearity parameter. Remarkably, two behaviors for deficit angle are seen for this case and no singularity takes place. There is an extremum $r_{0_{\mathrm{ext}}}$ in which for case of $r_{0} \leq r_{0_{\mathrm{ext}}}$, deficit angle 

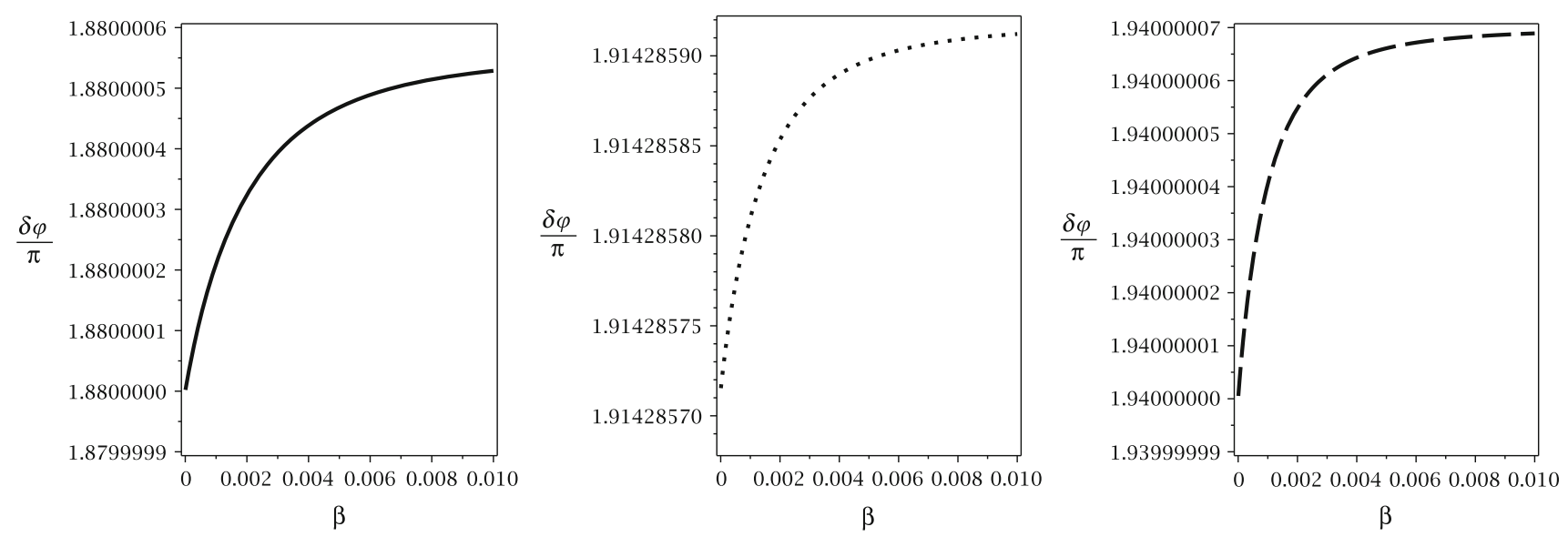

Fig. 9 LNED solutions: Different scales of $\delta \phi / \pi$ versus $\beta$ for $q=0.06$, and $l=0.3, r_{0}=5$ (continuous line), $r_{0}=7$ (dotted line) and $r_{0}=10$ (dashed line)

is a decreasing function of $r_{0}$ and for case of $r_{0} \geq r_{0_{\mathrm{ext}}}$, it is an increasing function of $r_{0}$. For large values of $r_{0}$ the effect of nonlinearity will decrease and obtained values of deficit angle for different cases of nonlinearity parameter will be so close. From Fig. 8 one can show that the highest and lowest values, in logarithmic form of nonlinear electrodynamics, of deficit angle is located at

$\left.\delta \varphi\right|_{\min }=\lim _{\beta \rightarrow 0} \delta \varphi$,

$\left.\delta \varphi\right|_{\max }=\lim _{\beta \rightarrow \infty} \delta \varphi$.

\subsection{Spining solutions}

Now, we would like to endow our spacetime solution (12) with a global rotation. In order to add an angular momentum to the spacetime, we perform the following local rotation boost in the $t-\varphi$ plane

$t \longmapsto \Xi t-a \varphi, \varphi \longmapsto \Xi \varphi-\frac{a}{l^{2}} t$,

where $a$ is the rotation parameter and $\Xi=\sqrt{1+a^{2} / l^{2}}$. Inserting Eq. (69) into Eq. (42) we obtain

$$
\begin{aligned}
\mathrm{d} s^{2}= & -\frac{r^{2}+r_{0}^{2}}{l^{2}}(\Xi \mathrm{d} t-a \mathrm{~d} \varphi)^{2} \\
& +\frac{r^{2}}{\left(r^{2}+r_{0}^{2}\right) g(r)} \mathrm{d} r^{2}+l^{2} g(r)\left(\frac{a}{l^{2}} \mathrm{~d} t-\Xi \mathrm{d} \varphi\right)^{2},
\end{aligned}
$$

where $g(r)$ is the same as that given in Eqs. (43), (44) and (46) for different NEDs. The nonzero components of the electromagnetic field are given as

$F_{r t}=-\frac{a}{\Xi l^{2}} F_{r \varphi}$

where Eq. (71) is valid for all mentioned models.
The local transformation (69) generates a new metric, because it is not a proper coordinate transformation on the entire manifold. Therefore, the metric (42) and (70) can be locally mapped into each other but not globally, and so they are distinct. It is notable to mention that $g(r)$ is always positive for $r>0$ and this spacetime has a conical singularity at $r=0$.

Now, we want to obtain the electric charge of the solutions. To determine the electric field, we should consider the projection of the electromagnetic field tensor on special hypersurface. The electric charge can be found by calculating the flux of the electric field at infinity, yielding

$Q=\frac{q}{2 \pi} \sqrt{\Xi^{2}-1} \times \begin{cases}2^{s-1} \mathrm{sq}^{2 s-2}, & \text { PMI } \\ 1, & \text { LNED, ENED }\end{cases}$

Notice that the electric charge is proportional to the rotation parameter, and is zero for the static spacetime. Finally, we calculate the conserved quantities of these solutions. The mass and the angular momentum of the magnetic solution can be calculated through the use of counterterm method. Using Eqs. (10) and (11), and the suitable counterterm Lagrangian $\mathcal{L}_{\mathrm{ct}}=1 / l$, one finds

$M=\frac{m}{8}\left(2 \Xi^{2}-1\right)$,

$J=\frac{\Xi m a}{4}$,

which Eq. (74) confirms that $a$ is rotation parameter.

\section{Nonlinearity as a correction}

It is arguable that instead of considering a theory which has the property of being highly nonlinear, one can add correction terms to the Maxwell theory in which we define as 
additional correction (AC). Regarding the weak field limit of nonlinear models, one can add quadratic Maxwell invariants to the Lagrangian of Maxwell theory. This consideration can be justified through following reasons. First of all, in series expanding BI types of nonlinear theories, the first leading term, which is coupled with nonlinearity parameter, is quadratic Maxwell invariant. Second, in low energy effective of string theory, this term could be obtained which gives strong motivation for considering this kind of modification. Third, in order to find experimental result for nonlinear theories one should take into account only small degrees of nonlinearity. Therefore, one can build another nonlinear theory of electromagnetic field [111-116].

The BI-type Lagrangians (20) and (26) for the large values of $\beta$ ( $\beta$ is nonlinearity parameter) tend to the following nonlinear Lagrangian

$\mathcal{L}_{A C}(\mathcal{F})=-\mathcal{F}+\alpha \mathcal{F}^{2}+O\left(\alpha^{2}\right)$,

where $\alpha$ is nonlinearity parameter and is proportional to the inverse value of nonlinearity parameter in BI-type theories, namely $\beta$. In Eq. (75), the nonlinearity parameter is small and so the effects of this parameter should be considered as a perturbation and in the limit $\alpha \longrightarrow 0, \mathcal{L}_{A C}(\mathcal{F})$ reduces to the Lagrangian of the standard Maxwell field, $\mathcal{L}_{\text {Maxwell }}(\mathcal{F})=$ $-\mathcal{F}$.

In this case, we want to obtain the solutions of Einstein gravity in presence of the nonlinear electrodynamics, which presented by the Lagrangian (75), for static and rotating metrics. As described in the previous sections, considering Eqs. (3), (12) and (75), one can show that

$$
\left(1-\frac{12 \alpha F_{\varphi \rho}^{2}}{l^{2}}\right) F_{\varphi \rho}^{\prime}+\left(1+\frac{4 \alpha F_{\varphi \rho}^{2}}{l^{2}}\right) \frac{F_{\varphi \rho}}{\rho}+O\left(\rho^{2}\right)=0,
$$

where Eq. (76) has the following solution

$F_{\varphi \rho}=\frac{q}{\rho}-\frac{4 q^{3} \alpha}{\rho^{3} l^{2}}+O\left(\alpha^{2}\right)$.

\section{Static AC magnetic solution}

To obtain the function $g(\rho)$, one can insert Eqs. (12) and (77) in the gravitational field equation (2) to obtain the metric function $g(\rho)$ as

$$
g(\rho)=m-\Lambda \rho^{2}+\frac{2 q^{2}}{l^{2}} \ln \left(\frac{\rho}{l}\right)+\frac{2 q^{4} \alpha}{l^{4} \rho^{2}}+O\left(\alpha^{2}\right),
$$

where $m$ is the integration constant which is related to the mass of solutions. One can show that the metric (12) with the metric function (78) has a singularity at $\rho=0$ by calculating the Kretschmann scalar as

$$
\begin{aligned}
R_{\mu \nu \lambda \kappa} R^{\mu \nu \lambda \kappa}= & 12 \Lambda^{2}-\frac{8 \Lambda q^{2}}{l^{2} \rho^{2}} \\
& -\frac{4 q^{4}(4 \Lambda \alpha-3)}{l^{4} \rho^{4}}-\frac{80 q^{6} \alpha}{l^{6} \rho^{6}}+O\left(\alpha^{2}\right) .
\end{aligned}
$$

From Eq. (79) it is obvious that Kretschmann scalar divergence at $\rho=0$ and reduces to $12 \Lambda^{2}$ for $\rho \longrightarrow \infty$. On the other hand, as mentioned before, because of changing in signature, it is not possible to extend spacetime to $\rho<r_{0}$. Also, one can apply the coordinate transformation (41) to the metric (12) and find the metric function as

$$
\begin{aligned}
g(\rho)= & m-\Lambda\left(r^{2}+r_{0}^{2}\right) \\
& +\frac{2 q^{2}}{l^{2}} \ln \left(\frac{\left(r^{2}+r_{0}^{2}\right)^{1 / 2}}{l}\right)+\frac{2 q^{4} \alpha}{l^{4}\left(r^{2}+r_{0}^{2}\right)}+O\left(\alpha^{2}\right),
\end{aligned}
$$

and the electromagnetic field in the new coordinate is

$F_{\varphi r}=\frac{q}{\left(r^{2}+r_{0}^{2}\right)^{1 / 2}}-\frac{4 q^{3} \alpha}{\left(r^{2}+r_{0}^{2}\right)^{3 / 2} l^{2}}+O\left(\alpha^{2}\right)$.

Since all curvature invariants do not diverge in the range $0 \leq r<\infty$, one finds that there is no essential singularity. But, like previous cases, this spacetime has a conical singularity at $r=0$ with the deficit angle $\delta \varphi=8 \pi \mu$ where $\mu$ is given by Eq. (54) and $\Omega$ has the following form

$\left.\Omega\right|_{A C}=4 l r_{0}\left[\Lambda-\left(\frac{q}{l r_{0}}\right)^{2}+2 \alpha\left(\frac{q}{l r_{0}}\right)^{4}\right]+O\left(\alpha^{2}\right)$.

In this case, the divergency of the deficit angle is located at

$\left.r_{0}\right|_{\delta \varphi \rightarrow \infty}=\frac{(2 \mathcal{X})^{\frac{1}{3}}+2 q+2 q^{2}\left(4 \mathcal{X}^{-1}\right)^{\frac{1}{3}}}{3 \Lambda l^{2}} q$,

$\mathcal{X}=q\left[4 q-27 \Lambda^{2} l^{2} \alpha+3 \Lambda l \sqrt{3 \alpha\left(27 \Lambda^{2} l^{2} \alpha-8 q^{2}\right)}\right]$,

and the root of the deficit angle is located at

$\left.r_{0}\right|_{\delta \varphi=0}=\frac{(9 \sqrt{2} \mathcal{Y})^{\frac{2}{3}} q+2 q^{2}\left(3 \mathcal{Y}^{\frac{1}{3}}+6^{\frac{2}{3}} q\right)}{9 l(\Lambda l+2) \mathcal{Y}^{\frac{1}{3}}}$,

where

$$
\begin{aligned}
\mathcal{Y}=q\left[\frac{4}{3} q^{2}-9 \alpha(\Lambda l+2)^{2}\right. & \\
& \left.+9(\Lambda l+2) \sqrt{\alpha\left[\alpha(\Lambda l+2)^{2}-\frac{8 q^{2}}{27}\right]}\right] .
\end{aligned}
$$



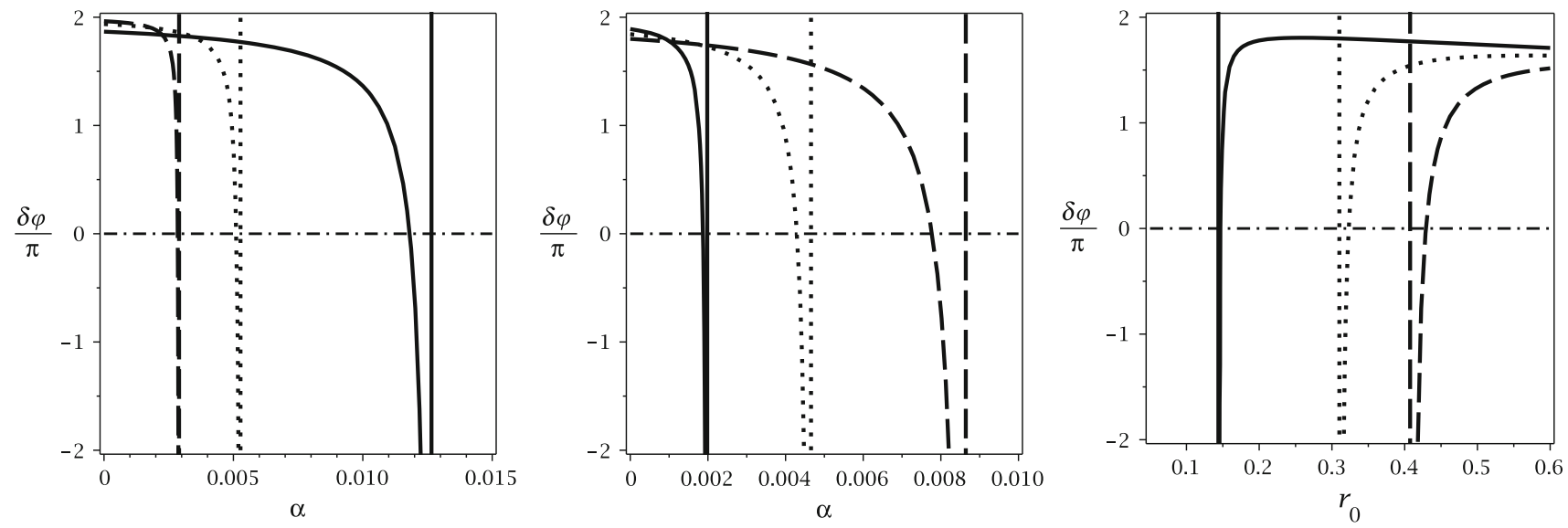

Fig. 10 AC solutions: $\delta \phi / \pi$ versus $\alpha$ (left and middle) and $\delta \phi / \pi$ versus $r_{0}($ right $)$ for $l=0.3$. Left diagram $r_{0}=1, q=2$ (continuous line), $q=3$ (dotted line) and $q=4$ (dashed line). Middle diagram $q=1$,

Considering the importance of deficit angle and its contribution to geometry of solutions, we plot various graphs (Fig. 10) for studying the effects of variation of parameters on deficit angle.

For the case of additional correction as nonlinear electromagnetic field, following effects were seen. As for the case of variation of charge (Fig. 10 left), deficit angle has a singularity. In other words, there is a divergency in which before and after that deficit angle is showing different behavior. Before divergency deficit angle is a decreasing function of nonlinearity parameter and there is a root for it and a region in which it has negative value. Whereas after divergency the deficit angle is a decreasing function of nonlinearity and it is always positive which is located in the region of non acceptable values. In other words, the values of the deficit angle after divergency are not in the upper bound limit of the deficit angle. The place of this divergency is a decreasing function of charge.

Next, as for the effects of $r_{0}$ Fig. 10 (middle) is plotted in which, there is a singularity for deficit angle. The place of divergency is an increasing function of the $r_{0}$. This behavior is opposite to the behavior of deficit angle for variation of electric charge.

The effects of variation of nonlinearity parameter is seen in Fig. 10 (right). Plotted graph for deficit angle versus $r_{0}$ shows that in essential, there is a divergency for calculated values of deficit angle. The place of this divergency is an increasing function of nonlinearity parameter which means that, as nonlinearity parameter increases, the place of this divergency will move to higher values of $r_{0}$. In case of deficit angle versus $r_{0}$, the behavior of system is quite different. Opposite to the previous case, in this plot before singularity, deficit angle is an increasing function of $r_{0}$ which is higher than upper bound limit for deficit angle and after that, there will be a region of negative deficit angle. This region and its related root are increasing functions of nonlinearity parameter. The $r_{0}=0.2$ (continuous line), $r_{0}=0.3$ (dotted line) and $r_{0}=0.4$ (dashed line). Right diagram $q=1, \alpha=0.001$ (continuous line), $\alpha=0.005$ (dotted line) and $\alpha=0.009$ (dashed line)

lowest value of deficit angle belongs to the highest value of nonlinearity parameter.

\section{Spinning AC magnetic solution}

In order to add angular momentum to the spacetime, we insert Eq. (69) into Eq. (42) and we obtain the rotating metric (70), where $g(r)$ is the same as $g(r)$ which is given in Eq. (80). The electromagnetic filed components become

$$
\begin{aligned}
F_{r t}= & -\frac{a}{\Xi l^{2}} F_{r \varphi}=\frac{a}{\Xi l^{2}}\left(\frac{q}{\left(r^{2}+r_{0}^{2}\right)^{1 / 2}}-\frac{4 q^{3} \alpha}{\left(r^{2}+r_{0}^{2}\right)^{3 / 2} l^{2}}\right) \\
& +O\left(\alpha^{2}\right) .
\end{aligned}
$$

The electric charge can be found by calculating the flux of the electric field at infinity, yielding

$Q=\frac{q}{2 \pi} \sqrt{\Xi^{2}-1}$.

On may note that the electric charge is proportional to the rotation parameter and for the static case $(\Xi=1)$ is zero. Also, one can show that the mass and the angular momentum of the solution are same as those in Eqs. (73) and (74), respectively.

At last, in order to obtain an insight regarding the negative deficit angle to geometrical structure of the solutions, we first give a description regarding positive deficit angle. The conic-like structure of the solutions is due to absence of specific value of angle that was cut from spacetime. This angle is deficit angle and has a positive value. In case of negative value of deficit angle, it is like an added segment to the spacetime. This adding will change the structure of the spacetime to a saddle-like cone (for more details see Fig. 2 in Ref. [117]). This negative deficit angle is known as surplus angle. It is worthwhile to mention that there is an upper 
bound for positive deficit angle whereas such bound does not exist for negative values of deficit angle.

\section{Conclusions}

In this paper, we considered different nonlinear theories of electrodynamics and study their three dimensional magnetic solutions. Although these theories of nonlinearity are generalization of Maxwell theory, in essence they are describing different phenomena. The obtained metric functions for these nonlinear theories showed quite different structures for magnetic solutions which in result enforcing their own conditions.

The primitive motivation of considering the mentioned metric was obtaining magnetic solutions through topological defects. In other words, the obtained values are representing topological defects. This conclusion is valid because of the geometrical structure of obtained solutions and the important property known as deficit angle. The deficit angle shows that the object that we are studying is not usual geometrical object. In case of obtained solutions in this paper, due to being three dimensional, their $t=$ cte and $r=$ cte geometry is a ring-like. Its shape and general properties such as area are described and determined by the value of deficit angle.

At first we focused on the energy density. Studying energy conditions in context of this spacetime, revealed the fact that PMI, LNED and ENED theories satisfy null, weak, strong and dominant energy conditions. In case of PMI theory, dominant energy conditions put a restriction on valid range of $s$ parameter $\left(\frac{1}{2}<s \leq 1\right)$. No restriction was observed for LNED and ENED. Next we studied the effects of different nonlinear theories on energy density and compare them with Maxwell theory. Interestingly for case of PMI theory, we had two sets of behavior. In general the energy density was an increasing function of $s$. Considering the fact that $s=1$ is denoted as Maxwell theory, we found that in case of $\frac{1}{2}<s<1(s>1)$ the concentration volume of energy density was smaller (larger) comparing to Maxwell theory. On the other hand, BI-types theories (LNED and ENED) had larger energy density than Maxwell theory. In general in these two theories energy density was a decreasing function of nonlinearity parameter. Therefore, considering the fact that for large values of nonlinearity parameter, these two theories reduce to Maxwell theory, one expect that the lowest energy density between these theories belongs to Maxwell theory, which is consistent with obtained results.

Here we are encountering another important difference between PMI theory and BI-types theories. In essence, the generalization of Maxwell theory to nonlinear theories of BI-types causes an increase in energy density. This increase indicates that the distribution of matter filed in these nonlinear theories is more concentrated comparing to Maxwell theory. On the other hand, for PMI theory two scenarios is possible. In one $\left(\frac{1}{2}<s<1\right)$, considering this nonlinear theory causes to decrease of energy density. In other words, in this case the distribution of matter filed is less concentrated comparing to Maxwell theory. On the other hand, for the one $(s>1)$, the energy density becomes larger comparing to Maxwell theory. This two different behavior is a unique characteristic of PMI theory and emphasizes the different nature of this theory from BI-types. If one consider dominant energy condition and its restrictions on theories of nonlinearity as dominant limitations, PMI theory only decreases the energy density whereas the BI-types increases the energy density. In the other words, these two classes of nonlinear theories have opposite effects on energy density.

In essence, PMI theory is a different theory comparing to other ones in conditions and evaluated values. The existence of $s$ as a power makes the magnetic solution related to it more sensitive to variation of $s$ comparing to variation of nonlinearity parameter in other theories. The places of deficit angel root and divergency were highly sensitive to variation of $s$. Due to structure of this theory two behaviors were seen for different values of $s$ which is a characteristic that only belongs to this theory. These different behaviors add another free parameter to this theory and make it possible to consider two approaches for studying magnetic solutions. It also states that in considering this theory, one must take this undeniable important property into consideration for studying solutions and their properties such as conserved quantities and their general behaviors.

In addition, this fact is of importance to mention that in usual charged three dimensional solutions, one expects the rise of logarithmic function of radial coordinate in metric function. This function was seen in BI-type nonlinear theories whereas for the case of PMI, interestingly, only for certain value of $s$ this function was seen. This fact emphasis another fundamental difference between this theory of nonlinear electromagnetic field and BI-type ones.

As for the AC theory, due to consideration of nonlinearity parameter as a correction to Maxwell theory, there was a restriction of considering only small values of nonlinearity parameter. Interestingly, in this theory, the existence of divergency was seen for deficit angle.

Remarkably, for case of LNED no singular point, hence no divergency was seen. Contrary to AC theory, this theory presented smooth and divergence free behavior for deficit angle. The obtained values of deficit angle for this theory were real and the only restriction that one may confront comes from the logarithmic part of solution which in plotted graphs for deficit angle no effect of this restriction was seen. Although both LNED and AC theories are in essence BI-types, this behavior is showing an important fact that they are in case of topological defects and magnetic solutions are describing completely different phenomena and they are independent 
of each other. The same property was seen for the case of exponential form.

It is notable to mention the fact that in plotted graphs of Maxwell, no singularity was seen. In fact, calculated values of divergence point showed that there are two divergence points that in AdS spacetime they are not real. In other words, in case of AdS spacetime, deficit angle is divergence free. Opposite to the case of divergency, we found a relation for roots in this case which indicated three different possible cases: two roots, one extreme root and no root.

One of the important issues that must be taken into consideration is the existence of roots for deficit angle. The existence of root for deficit angle states that no contributing to structure of magnetic solution exists. In other words, the object that we are studying in these special cases are not cosmological (topological) defects and they do not have the property of being cosmological defects. If we consider the cosmological defects as dynamic objects that their parameters may vary through time, one may say that for special values of parameters, the object will change into another astrophysical object (no deficit angle is seen). But this idea is only acceptable if the root of deficit angle is extreme or the region in which deficit angle is negative.

Also, the existence of negative values of deficit angle poses another important issue. The structure of magnetic solution and the meaning of having negative deficit angle is something that must be taken into consideration and studied in more details.

One may interpret that roots of deficit angle may present the phase transition for these astrophysical objects and the negative values of deficit angle are representing another phase for them. Or one may say that negative and positive values of deficit angle are representing two different types of defects. The roots are places where these phase transitions take place. Considering the fact that in calculation of deficit angle, one is using second order derivation of metric function with respect to radial coordinate (see for example chapter 9 of Ref. [118]) and if one consider the metric function as a potential, it is arguable that the roots of deficit angle are representing phase transition. On the other hand, considering the concept of divergency of potential as a point of phase transition, one may argue that existence of divergency in deficit angle is representing phase transition. Therefore, one may state that instead of taking roots of deficit angle as phase transition points, singular points must be taken into consideration as phase transition points. These phase transitions may be geometrical types of transitions. In other words, the shape of the object may only change, not its physical being change into another thing. But this idea is debatable if one consider roots of deficit angle as phase transition. It is due to fact that topological property which describe the shape of the magnetic object will be quite different before and after phase transition in which the sign of deficit angle will change. In some of the nonlinear theories and Maxwell one no singularity was seen which state that in concept of considering divergency as a phase transition, these theories are in fact without phase transition. But as it was mentioned before, in case of Maxwell theory, the background spacetime (AdS/dS) plays the crucial role. In AdS spacetime there is no divergency and for dS spacetime one can find divergence points and therefore it may have phase transition. But if one consider roots as phase transition in both spacetime, phase transitions take place.

Another interesting issue comes from studying Fig. 4. In the absence of charge, $q=0$, the deficit angle could be non zero. By adding charge to solutions and increasing it, the deficit angle increases and general behavior of it is also modified. This shows the fact that contribution of charge to deficit angle is of an increasing factor. In other words, electromagnetic field will increase the value of deficit angle.

Finally it is quite important to mention the fact that the only non zero component of considered gauge potential in case of these topological defects were spatial one which was considered as provider of magnetic field. By applying the mentioned transformation and changing metric from static to rotating one, another component was added to electromagnetic field tensor which was the well-known provider of electric field. Obtained values for this electric part of electromagnetic field tensor were functions of rotating parameter and in case of setting rotating parameter equal to zero, these electric field would vanish. This fact shows that obtained values are essentially magnetic solutions.

Acknowledgments We thank Shiraz University Research Council. This work has been supported financially by Research Institute for Astronomy and Astrophysics of Maragha.

Open Access This article is distributed under the terms of the Creative Commons Attribution 4.0 International License (http://creativecomm ons.org/licenses/by/4.0/), which permits unrestricted use, distribution, and reproduction in any medium, provided you give appropriate credit to the original author(s) and the source, provide a link to the Creative Commons license, and indicate if changes were made. Funded by SCOAP ${ }^{3}$.

\section{References}

1. A. Mesaros et al., Science 333, 426 (2011)

2. G.P. Alexander, B.G. Chen, E.A. Matsumoto, R.D. Kamien, Rev. Mod. Phys. 84, 1229 (2012)

3. M. Mielenz et al., Phys. Rev. Lett. 110, 133004 (2013)

4. U. Tkalec et al., Science 333, 6038 (2011)

5. N.D. Mermin, Rev. Mod. Phys. 51, 591 (1979)

6. H. Braun, Adv. Phys. 61, 1 (2012)

7. D.M. Stamper-Kurn, M. Ueda, Rev. Mod. Phys. 85, 1191 (2013)

8. G. Aad et al., Phys. Rev. D 90, 052004 (2014)

9. T. Simula, Phys. Rev. A 87, 023630 (2013)

10. J. Lovegrove, M.O. Borgh, J. Ruostekoski, Phys. Rev. Lett. 112, 075301 (2014) 
11. J. Sabbatini, W.H. Zurek, M.J. Davis, Phys. Rev. Lett. 107, 230402 (2011)

12. N. Navon, A.L. Gaunt, R.P. Smith, Z. Hadzibabic, Science 347, 167 (2015)

13. J. Kierfeld, T. Nattermann, T. Hwa, Phys. Rev. B 55, 626 (1997)

14. E. Babaev, Nucl. Phys. B 686, 3 (2004)

15. J.C.Y. Teo, C.L. Kane, Phys. Rev. B 82, 115120 (2010)

16. A. Moor, A.F. Volkov, K.B. Efetov, Phys. Rev. B 90, 224512 (2014)

17. R.H. Brandenberger, Int. J. Mod. Phys. A 09, 2117 (1994)

18. T.W.B. Kibble, J. Phys. A 09, 1387 (1976)

19. A. Guth, Phys. Rev. D 23, 347 (1980)

20. T.W.B. Kibble, Phys. Rep. 67, 183 (1980)

21. A. Vilenkin, Phys. Rep. 121, 263 (1985)

22. N. Turok, Phase transition as the origin of large-scale structure. in Particles, Strings and Supernovae (TASI-88), eds. by A. Jevicki, C.I. Tan (World Scientific, Singapore, 1989)

23. A. Vilenkin, E.P.S. Shellard, Topological Defects and Cosmology (Cambridge University Press, Cambridge, 1994)

24. R.H. Brandenbergera, A. Davis, Phys. Lett. B 322, 3 (1994)

25. O.J.C. Dias, J.P.S. Lemos, Class. Quant. Gravit. 19, 2265 (2002)

26. M.H. Dehghani, Phys. Rev. D 69, 044024 (2004)

27. M.H. Dehghani, Phys. Rev. D 70, 064019 (2004)

28. E. Witten, Nucl. Phys. B 249, 557 (1985)

29. I. Moss, S. Poletti, Phys. Lett. B 199, 34 (1987)

30. P. Peter, Phys. Rev. D 49, 5052 (1994)

31. C.N. Ferreira, M.E.X. Guimaraes, J.A. Helayel-Neto, Nucl. Phys. B 581, 165 (2000)

32. M.H. Dehghani, Phys. Rev. D 71, 064010 (2005)

33. A. Sheykhi, Phys. Lett. B 672, 101 (2009)

34. A.A. Sen, Phys. Rev. D 60, 067501 (1999)

35. R.H. Brandenberger, A.C. Davis, A.M. Matheson, M. Trodden, Phys. Lett. B 293, 287 (1992)

36. M. Giovannini, Lect. Notes Phys. 737, 863 (2008)

37. L.V. Zadorozhna, B.I. Hnatyk, Y.A. Sitenko, UJP 58, 398 (2013)

38. L. Del Debbio, A. Di Giacomo, Y.A. Simonov, Phys. Lett. B 332, 111 (1994)

39. H.G. Dosch, O. Nachtmann, M. Rueter. arXiv:hep-ph/9503386

40. A. Gorsky, V. Zakharov, Phys. Rev. D 77, 045017 (2008)

41. Y.A. Sitenko, Nucl. Phys. B 372, 622 (1992)

42. M. Bordag, S. Voropaev, Phys. Lett. B 333, 238 (1994)

43. M.A. Giorgi, Int. J. Mod. Phys. A 20, 5069 (2005)

44. K. Skenderis, P.K. Townsend, A.V. Proeyen, JHEP 08, 036 (2001)

45. J.C.R.E. Oliveira, C.J.A.P. Martins, P.P. Avelino, Phys. Rev. D 71, 083509 (2005)

46. D.P. George, M. Trodden, R.R. Volkas, JHEP 02, 035 (2009)

47. N.G. Phillips, A. Kogut, Phys. Rev. Lett. 75, 1264 (1995)

48. I. Cho, Phys. Rev. D 66, 045028 (2002)

49. M.V. Sazhin et al., Mon. Not. R. Astron. Soc. 376, 1731 (2007)

50. G. Clement, Class. Quant. Grav. 10, L49 (1993)

51. E.W. Hirschmann, D.L. Welch, Phys. Rev. D 53, 5579 (1996)

52. M. Cataldo, P. Salgado, Phys. Rev. D 54, 2971 (1996)

53. O.J.C. Dias, J.P.S. Lemos, JHEP 01, 006 (2002)

54. M. Cataldo, J. Crisostomo, S. del Campo, P. Salgado, Phys. Lett. B 584, 123 (2004)

55. W.T. Kim, D. Park, J. Korean Phys. Soc. 55, 389 (2009)

56. D.H. Delphenich, Nonlinear electrodynamics and QED. arXiv:hep-th/0309108

57. D.H. Delphenich, Nonlinear optical analogies in quantum electrodynamics. arXiv:hep-th/0610088

58. W. Heisenberg, H. Euler, Z. Phys. 98, 714 (1936). arXiv:physics/0605038(Translation by: W. Korolevski, H. Kleinert, Consequences of Dirac's Theory of the Positron)

59. J. Schwinger, Phys. Rev. 82, 664 (1951)

60. H. Yajima, T. Tamaki, Phys. Rev. D 63, 064007 (2001)

61. V.A. De Lorenci, M.A. Souza, Phys. Lett. B 512, 417 (2001)
62. V.A. De Lorenci, R. Klippert, Phys. Rev. D 65, 064027 (2002)

63. M. Novello et al., Class. Quant. Gravit. 20, 859 (2003)

64. M. Novello, E. Bittencourt, Phys. Rev. D 86, 124024 (2012)

65. E. Ayon-Beato, A. Garcia, Gen. Relat. Gravit. 31, 629 (1999)

66. E. Ayon-Beato, A. Garcia, Phys. Lett. B 464, 25 (1999)

67. V.A. De Lorenci, R. Klippert, M. Novello, J.M. Salim, Phys. Rev. D 65, 063501 (2002)

68. I. Dymnikova, Class. Quant. Gravit. 21, 4417 (2004)

69. C. Corda, H.J. Mosquera Cuesta, Mod. Phys. Lett A 25, 2423 (2010)

70. C. Corda, H.J. Mosquera, Cuesta. Astropart. Phys. 34, 587 (2011)

71. Z. Bialynicka-Birula, I. Bialynicka-Birula, Phys. Rev. D 2, 2341 (1970)

72. H.J. Mosquera, J.M. Cuesta Salim, Mon. Not. R. Astron. Soc. 354, L55 (2004)

73. H.J. Mosquera, J.M. Cuesta Salim, Astrophys. J. 608, 925 (2004)

74. M. Born, L. Infeld, Proc. R. Soc. A 144, 425 (1934)

75. H.H. Soleng, Phys. Rev. D 52, 6178 (1995)

76. S.H. Hendi, JHEP 03, 065 (2012)

77. M. Hassaine, C. Martinez, Phys. Rev. D 75, 027502 (2007)

78. M. Hassaine, C. Martinez, Class. Quant. Gravit. 25, 195023 (2008)

79. S.H. Hendi, H.R. Rastegar-Sedehi, Gen. Relativ. Gravit. 41, 1355 (2009)

80. S.H. Hendi, Phys. Lett. B 677, 123 (2009)

81. H. Maeda, M. Hassaine, C. Martinez, Phys. Rev. D 79, 044012 (2009)

82. S.H. Hendi, B. Eslam, Panah. Phys. Lett. B 684, 77 (2010)

83. G. Boillat, J. Math. Phys. (N.Y.) 11, 941 (1970)

84. G. Boillat, J. Math. Phys. (N.Y.) 11, 1482 (1970)

85. G.W. Gibbons, D.A. Rasheed, Nucl. Phys. B 454, 185 (1995)

86. Z. Zhao, Q. Pan, S. Chen, J. Jing, Nucl. Phys. B 871, 98 (2013)

87. V. Brindejonc, Class. Quant. Gravit. 15, 3599 (1998)

88. C. Martinez, C. Teitelboim, J. Zanelli, Phys. Rev. D 61, 104013 (2000)

89. E. Ayón-Beato, A. Garbarz, G. Giribet, M. Hassaine, Phys. Rev. D 80, 104029 (2009)

90. S.H. Hendi, Eur. Phys. J. C 69, 281 (2010)

91. F. Darabi, K. Atazadeh, A. Rezaei-Aghdam, Eur. Phys. J. C 73, 2657 (2013)

92. M. Hassaine, Phys. Rev. D 89, 044009 (2014)

93. S.H. Hendi, B. Panah Eslam, R. Saffari, Int. J. Mod. Phys. D 23, 1450088 (2014)

94. S. Carlip, Class. Quant. Gravit. 12, 2853 (1995)

95. A. Ashtekar, J. Wisniewski, O. Dreyer, Adv. Theor. Math. Phys. 6, 507 (2002)

96. T. Sarkar, G. Sengupta, B.N. Tiwari, JHEP 11, 015 (2006)

97. E. Witten, Adv. Theor. Math. Phys. 2, 505 (1998)

98. S. Carlip, Class. Quant. Gravit. 22, R85 (2005)

99. E. Witten. arXiv:0706.3359

100. S.H. Mazharimousavi, M. Halilsoy, O. Gurtug, Eur. Phys. J. C 74, 2735 (2014)

101. S.H. Mazharimousavi, M. Halilsoy, Eur. Phys. J. Plus, 130, 158 (2015)

102. R.C. Myers, Phys. Rev. D 36, 392 (1987)

103. S.C. Davis, Phys. Rev. D 67, 024030 (2003)

104. J. Maldacena, Adv. Theor. Math. Phys. 2, 231 (1998)

105. E. Witten, Adv. Theor. Math. Phys. 2, 253 (1998)

106. P. Kraus, F. Larsen, R. Siebelink, Nucl. Phys. B 563, 259 (1999)

107. O. Aharony, S.S. Gubser, J. Maldacena, Y. Oz, Phys. Rept. 323, $183(2000)$

108. M.H. Dehghani, R.B. Mann, Phys. Rev. D 64, 044003 (2001)

109. M.H. Dehghani, Phys. Rev. D 65, 104030 (2002)

110. J.D. Brown, J.W. York, Phys. Rev. D 47, 1407 (1993)

111. S.H. Hendi, Eur. Phys. J. C 73, 2634 (2013)

112. S.H. Hendi, S. Panahiyan, Phys. Rev. D 90, 124008 (2014) 
113. S.H. Hendi, M. Momennia, Eur. Phys. J. C 75, 54 (2015)

114. S.H. Hendi, Int. J. Mod. Phys. D 24, 1550040 (2015)

115. S.H. Hendi, B. Panah Eslam, S. Panahiyan, Phys. Rev. D 91, 084031 (2015)

116. S.H. Hendi, S. Panahiyan, B. Panah Eslam, Eur. Phys. J. C 75, 296 (2015)
117. C. de Rham, Living Rev. Relativ. 17, 7 (2014)

118. R. d'Inverno, Introducing Einstein's Relativity (Oxford University Press Inc., New York, 1996) 